\title{
The effectiveness of peer-supported interventions for encouraging dietary behaviour change in adults: a systematic review
}

\author{
Sarah E Moore*, Megan McMullan, Claire T McEvoy, Michelle C McKinley and \\ Jayne $\mathrm{V}$ Woodside \\ Nutrition Research Group, Centre for Public Health, Queen's University Belfast, Institute of Clinical Science A, \\ Grosvenor Road, Belfast BT12 6BJ, UK
}

Submitted 12 December 2017: Final revision received 8 October 2018: Accepted 22 October 2018: First published online 3 December 2018

\begin{abstract}
Objective: There is an urgent need to find effective methods of supporting individuals to make dietary behaviour changes. Peer-supported interventions (PSI) have been suggested as a cost-effective strategy to support chronic disease selfmanagement. However, the effect of PSI on dietary behaviour is unclear. The present systematic review aimed to assess the effectiveness of PSI for encouraging dietary behaviour change in adults and to consider intervention characteristics linked with effectiveness.

Design: Electronic databases were searched until June 2018 for randomised controlled trials assessing the effectiveness of PSI compared with an alternative intervention and/or control on a dietary related outcome in adults. Following title and abstract screening, two reviewers independently screened full texts and data were extracted by one reviewer and independently checked by another. Results were synthesised narratively.

Setting: Randomised controlled trials.

Participants: Adult studies.

Results: The fifty-four included studies varied in participants, intervention details and results. More PSI reported a positive or mixed effect on diet than no effect. Most interventions used a group model and were lay-led by peer supporters. Several studies did not report intervention intensity, fidelity and peer training and support in detail. Studies reporting positive effects employed more behaviour change techniques (BCT) than studies reporting no effect; however, heterogeneity between studies was considerable.

Conclusions: As evidence was mixed, further interventions need to assess the effect of PSI on dietary behaviour, describe intervention content (theoretical basis, BCT, intensity and peer training/support) and include a detailed process evaluation.
\end{abstract}

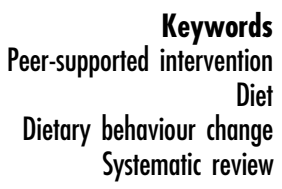

Strong evidence suggests that consumption of a healthy diet can reduce chronic disease risk ${ }^{(1)}$, but there is an urgent need to find effective methods of supporting individuals to make and sustain healthy dietary behaviour change. Lifestyle interventions encouraging dietary behaviour change are often intensive and expensive to roll out through health-care systems. Hence, there is increasing interest in the use of peer-supported interventions (PSI) as a potential flexible, cost-effective and more scalable strategy for improving health behaviours ${ }^{(2)}$. PSI have been shown to be an effective strategy for supporting selfmanagement of chronic diseases, such as diabetes ${ }^{(3,4)}$, and improvement of health behaviours, including physical activity $^{(5,6)}$; however, the effect of PSI on dietary behaviour change is not yet clear.

Findings from intervention studies are inconsistent, largely owing to heterogeneity between studies in terms of populations studied, PSI models and delivery ${ }^{(7)}$. There is no widely accepted definition of a PSI, which has led to broad interpretations of its meaning. PSI are typically delivered by lay individuals or community health workers (CHW), as opposed to health professionals, and these individuals may assume different roles including a peer supporter, peer educator, peer counsellor, peer facilitator or peer case manager ${ }^{(8)}$. PSI can be delivered via different models, including group, dyadic or a combination, and via 
face-to-face, telephone or the web. A recent systematic review suggested that a dyadic PSI model may be more effective in encouraging behaviour change than a groupbased model or a combination of dyadic and group-based support $^{(8)}$. Current literature, however, does not clearly describe an optimal PSI model, peer role, or the attributes, skills and training and support requirements needed to effectively undertake a peer role, and there is limited information on measurement of fidelity of PSI delivery. While optimal PSI durations have been suggested for improving chronic disease self-management ${ }^{(4)}$ and physical activity ${ }^{(6)}$, the duration of PSI needed to effectively encourage dietary behaviour change is not known. PSI to improve health behaviour have included various population groups ${ }^{(8)}$; however, it is not clear if PSI may be more effective in encouraging behaviour change among specific groups within the adult population. Additionally, previous research has not considered the impact of behaviour change theory/techniques used on PSI effectiveness ${ }^{(5,8)}$. Behaviour change theory and techniques are a key element of behaviour change interventions, and it has been demonstrated that the theories and techniques used could impact the effectiveness of behaviour change interventions ${ }^{(9)}$. Information on these PSI characteristics is therefore needed to guide the development of PSI targeting dietary behaviour change for public health.

Previous systematic reviews have examined the effect of PSI on health behaviours ${ }^{(5,8)}$ but, to our knowledge, none have focused on dietary behaviour change in the general adult population. An evaluation of existing evidence is required to determine the effectiveness of PSI for encouraging dietary behaviour change in adults, which in turn will help to inform the design of future studies and eventual public health policy and practice. Therefore, the present systematic review aimed to examine randomised controlled trials that measure the effectiveness of PSI on dietary outcomes in adults, in comparison with other types of intervention and/or a control, and to consider intervention characteristics that may be linked with effectiveness including PSI model used, peer role, peer attributes, behaviour change theory/techniques used, intervention duration and intensity, and population studied.

\section{Methods}

The review protocol is registered on PROSPERO (www. crd.york.ac.uk/PROSPERO/; identification number CRD42014009994). Subsequent to protocol registration, it was decided to include all types of PSI rather than peer support alone to enable comparison of effectiveness of interventions by peer role. PSI were described according to definitions provided in a recently conducted review ${ }^{(8)}$. This section reports the review protocol according to
PRISMA (Preferred Reporting Items for Systematic Reviews and Meta-Analyses) guidelines ${ }^{(10)}$.

\section{Search strategy}

A search strategy was devised considering key search terms used in previous PSI or dietary related reviews. A structured search using the devised strategy was then conducted in five electronic databases: MEDLINE, EMBASE, PsycINFO, CINAHL and the Cochrane Library. Databases were searched from inception until 14 June 2018. The search strategy consisted of subject headings and keyword terms relating to PSI, which were combined with terms relating to dietary behaviour change. The search strategy developed in MEDLINE is presented as an example (see online supplementary material, Table S1). This strategy was tailored for other databases. Searches were limited to those published in English language, human studies and adult studies in all databases where possible. Applying an age limit can lead to loss of studies that are not yet coded in the database by age. To assess the impact of this limit, the search was limited for each age limit in each database and run selecting to 'not' include these limits, leaving studies not coded by age. A significant number of studies were not coded by age in EMBASE; therefore, the search was rerun in this database without limiting for age.

Studies obtained were screened for suitability for inclusion in the review using the following inclusion and exclusion criteria, which were defined a priori.

\section{Inclusion criteria}

1. Adult subjects.

2. Assessed effectiveness of a PSI on dietary behaviour change in comparison with other interventions and/or a control.

3. Dietary or weight-related primary or secondary outcome. Dietary outcomes: any measurement of diet such as estimated intake, change in intake of energy, nutrients or food groups, or biomarker data. Weightrelated outcomes: weight, BMI, waist circumference and waist-to-hip ratio. While weight-related outcomes are an indirect indication of dietary behaviour, few randomised controlled trials have assessed dietary behaviour change outcomes, so inclusion of anthropometric measures provided useful additional information.

4. Randomised controlled trial, to determine whether a cause-and-effect relationship between PSI and dietary or weight-related outcomes exists. This study design was selected as it is the gold standard method for assessing the effect of interventions. Other study designs such as quasi-experimental studies were excluded because, due to lack of randomisation, systematic differences between intervention groups 
are not eliminated at the outset which weakens the ability to determine causality.

5. As the length of time needed to establish dietary behaviour change is not clear, interventions of all durations and lengths of follow-up were included.

\section{Exclusion criteria}

1. Studies targeting pregnant women, eating disorders or malnutrition.

2. Studies considering multiple behaviours where the effects of the intervention on diet could not be determined.

3. Multicomponent interventions where the effects of the PSI could not be determined.

4. Studies where it was unclear if the intervention was peer-led.

5. Interventions that included a peer-led component but were primarily professionally led.

6. Web interventions that did not have an interactive/ tailored component.

Initially, titles of studies were removed where it was clear that they would not be relevant for inclusion. Abstracts of potentially relevant titles were then obtained and screened. This process was conducted by the principal reviewer, due to the large volume of studies obtained. Full texts of potentially relevant abstracts were then obtained and were all screened independently by two researchers, with discrepancies resolved through discussion.

\section{Data extraction}

Data extraction was undertaken by the principal reviewer using a data extraction form, which was developed for the review and pilot tested on a small sample of studies ( $n$ 5) to ensure the desired data were captured. Data extraction was independently checked by another reviewer, with discrepancies between reviewers being resolved through discussion. Extracted data included participation details (number in analysis, gender, age, geographical region and characteristics), intervention details (intervention groups and outcome measure) and PSI details (content, behaviour change techniques (BCT) and theoretical framework used, model, peer, peer role, peer training and support, fidelity of PSI delivery and duration/intensity) and results (effect of PSI $v$. other intervention and/or control). BCT are strategies used to facilitate behaviour change and are therefore key components of dietary behaviour change interventions. BCT used in each PSI were extracted to provide insight into intervention characteristics associated with effectiveness of PSI to encourage dietary behaviour change. BCT were identified using a forty-item taxonomy of BCT to aid dietary behaviour change ${ }^{(11)}$. BCT used by both the experimental intervention and the comparison intervention were not included. To enable comparison of studies, PSI details, including intensity, model and peer roles, were extracted and categorised as suggested by a recent review of peer-led interventions ${ }^{(8)}$. As a measure of PSI intensity, it was recorded if the PSI was: (i) a single intervention; (ii) an intervention with multiple time points; or (iii) an ongoing intervention. PSI models were recorded as group, dyadic or hybrid (group and dyadic). Peer roles were defined as one or more of the following: (i) peer case manager (helps individuals access or coordinate health and social services including referral to resources, or managing intervention activities); (ii) peer counsellor (provides knowledge, guidance and tools to help individuals set and reach their health goals); (iii) peer educator (delivers formal education utilising a protocolled curriculum and approach); (iv) peer facilitator (facilitates group interactions to create or strengthen relationships to help individuals set and reach goals together); and (v) peer support (informal, unstructured support such as providing reminders, encouragement or reinforcement, informal coaching, and sharing personal experiences or narrative) $)^{(8)}$.

Additionally, risk of bias was assessed at the study level by the principal reviewer to aid with interpretation of the strength of findings. The JADAD scale ${ }^{(12)}$, a widely used quality assessment tool due to its simplicity, reliability and validity, was utilised. The scale assesses randomisation, blinding and participant follow-up. Points were awarded if: (i) the study was described as randomised; (ii) the method used to generate the sequence of randomisation was described and appropriate; (iii) the method of double blinding was described; (iv) the method of double blinding was appropriate; and (v) the number of and reasons for withdrawals were stated. Based on the JADAD score range obtained, methodological quality was categorised as low (0-1 points), moderate ( 2 points) or high ( $3-5$ points).

\section{Data synthesis}

Due to the heterogeneity of included studies, a narrative synthesis of results was undertaken ${ }^{(13)}$. A descriptive overview is provided of included studies, which includes information extracted on participation details, intervention details and PSI details. Findings were synthesised on the effect of PSI on dietary behaviour change outcomes, specifically if PSI were more effective than alternative methods of support. This was synthesised for all studies and then by type of dietary outcome measure used (dietary or anthropometric). Relationships in the data were explored through looking at the effect of PSI characteristics on dietary behaviour change including PSI model used, peer type/role used, behaviour change theory/ techniques used, PSI duration/intensity and population studied. Specifically, this was done through comparison of these characteristics in studies reporting that PSI were more effective than alternative methods, or reporting 
mixed results, with studies that found no significant difference between PSI and control groups. This was explored initially in all studies and then by type of dietary outcome used. To help ensure a robust analysis was conducted, the methodological quality of included studies was assessed $^{(12)}$ and the results of high-quality studies on the effect of PSI on dietary behaviour change outcome(s) were compared with the overall results from all studies on the effect of PSI on dietary behaviour change outcome(s), to confirm that they were consistent.

\section{Results}

\section{Effect of peer-supported interventions on dietary behaviour change}

A total of fifty-four studies were included within the review $^{(14-67)}$ (Fig. 1). The effect of PSI on dietary behaviour change outcomes varied (Table 1). In comparison with alternative methods of support or a control, fifteen studies (28\%) reported that PSI were more effective for encouraging dietary behaviour change, seventeen (31\%)

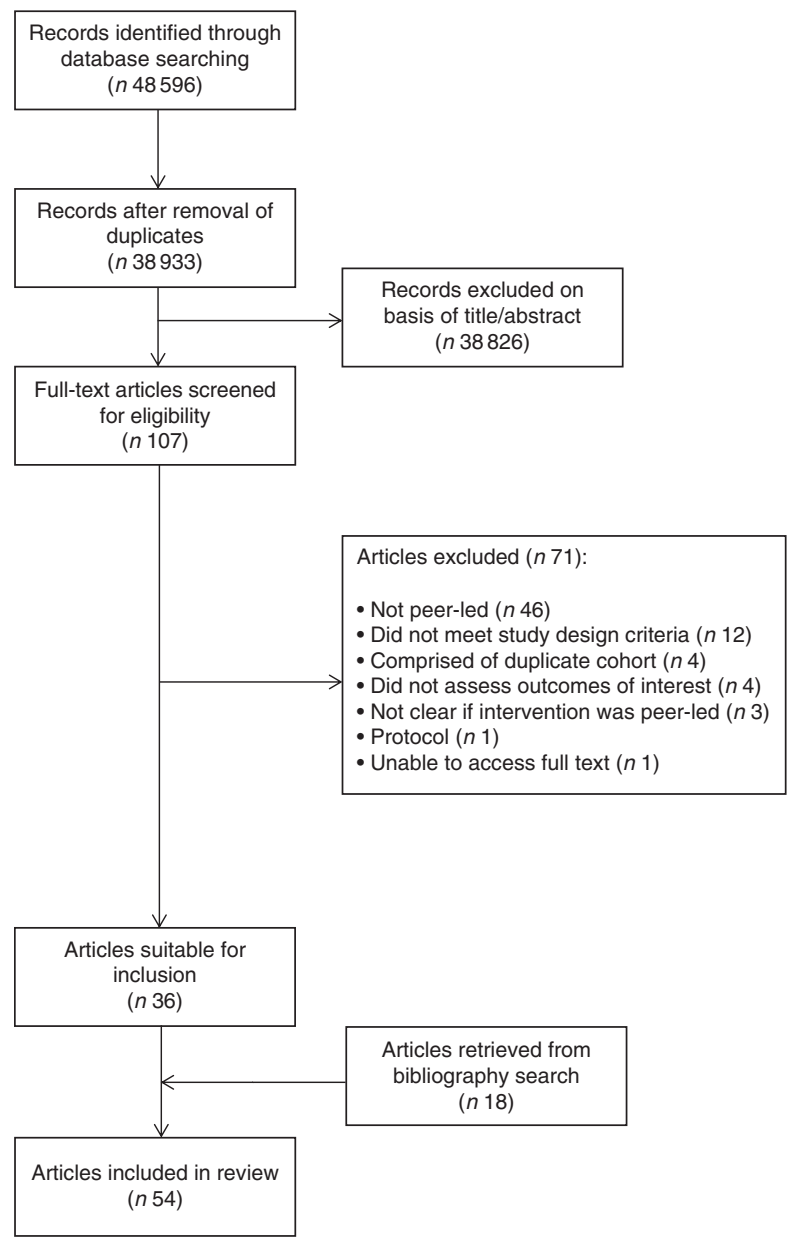

Fig. 1 Summary of study selection process for the present systematic review on the effectiveness of peer-supported interventions for encouraging dietary behaviour change in adults reported that there were no significant differences between methods, six (11\%) reported mixed results within each dietary behaviour change outcome measured (i.e. different results for different intervention groups/time points) and sixteen (30\%) reported mixed results between dietary behaviour change outcomes measured (i.e. different results for different dietary outcome measures in studies measuring more than one dietary outcome measure). To consider these findings further, studies were categorised into subsets based on outcome(s) used. Outcomes included dietary pattern ( $n 11)$, fruit and vegetable intake ( $n$ 17), fat intake ( $n$ 18), intake of other nutrients/foods ( $n$ 13), weight ( $n$ 27), BMI ( $n$ 23), and waist and hip measurements ( $n$ 18).

A total of thirty-five studies assessed the effect of PSI on dietary outcomes (Table 2). Eleven studies assessed the effect of PSI on overall dietary pattern. Four (36\%) of these studies used questionnaires to measure diet that were not validated for the study population. Just over half of the studies assessing dietary patterns reported that there was no significant difference between PSI and a control for improving diet ( $n$ 6; 55\%). In total, seventeen studies assessed the effect of PSI on fruit and vegetable intake. Most used validated measures of fruit and vegetable assessment including food diaries, FFQ and specific questions on diet. Five studies (29\%) reported that PSI were more effective than alternative interventions and/or a control for improving fruit and vegetable intake. Ten studies (59\%) reported no significant difference between PSI and alternative interventions and/or a control for improving fruit and vegetable intake. The remaining two studies (12\%) reported mixed results. Eighteen studies examined the effect of PSI on fat intake. Most used validated measures including food diaries, FFQ, dietary recalls and specific questions on diet. Eight studies (44\%) reported that PSI were more effective than a control for improving fat intake, eight studies (44\%) found no significant difference between PSI and an alternative intervention and/or a control for improving fat intake, and two studies (11\%) reported mixed results. Thirteen studies assessed the effect of PSI on intake of other nutrients/ foods, including energy, protein, carbohydrates, starch, fibre, sugar, sugar-sweetened beverages, non-sugar sweetened beverages, salt and fast foods. Most studies used validated measures to assess intakes of these nutrients including food diaries, FFQ, dietary recalls and specific questions on diet. One study out of thirteen (8\%) reported that a PSI was more effective than a control for reducing salt intake. Eight studies out of thirteen (62\%) reported no significant difference in intake of various nutrients between PSI and alternative interventions and/or a control. The remaining four studies (31\%) reported mixed results.

A total of forty studies assessed the effect of PSI on anthropometric outcomes. Anthropometric data were objectively measured in these studies; however, methods 
Table 1 Summary of the effect of peer-supported interventions (PSI) on dietary behaviour change outcomes $(n$ 54)

\begin{tabular}{|c|c|c|c|c|c|}
\hline \multirow[b]{2}{*}{ Study } & \multirow[b]{2}{*}{$\begin{array}{l}\text { Dietary behaviour change } \\
\text { outcome(s) measured }\end{array}$} & \multicolumn{4}{|c|}{ Effect of PSI } \\
\hline & & $\begin{array}{l}\text { PSI more } \\
\text { effective than } \\
\text { alternative }\end{array}$ & $\begin{array}{l}\text { No significant } \\
\text { difference between } \\
\text { PSI and control }\end{array}$ & $\begin{array}{l}\text { Mixed within } \\
\text { each } \\
\text { outcome* }^{*}\end{array}$ & $\begin{array}{c}\text { Mixed } \\
\text { between } \\
\text { outcomes† }\end{array}$ \\
\hline Garrett et al. (2005) $)^{(28)}$ & Dietary pattern & $\checkmark$ & & & \\
\hline Robinson-Whelen et al. (2006) ${ }^{(32)}$ & Dietary pattern & $\checkmark$ & & & \\
\hline Resnicow et al. (2004) & FV & $\checkmark$ & & & \\
\hline Campbell et al. (1999) & FV & $\checkmark$ & & & \\
\hline Toobert et al. $(2007)^{(35)}$ & Fat & $\checkmark$ & & & \\
\hline West et al. $(2011)^{(26)}$ & Weight & $\checkmark$ & & & \\
\hline Ackermann et al. $(2015)^{(29)}$ & Weight & $\checkmark$ & & & \\
\hline Lutes et al. (2017) & Weight & $\checkmark$ & & & \\
\hline Safford et al. $(2015)^{(52)}$ & $\mathrm{BMI}$ & $\checkmark$ & & & \\
\hline Katula et al. $(2013)^{(61)}$ & $\begin{array}{l}\text { Multiple weight (weight, BMI, } \\
\text { WC) }\end{array}$ & $\checkmark$ & & & \\
\hline Block et al. $(2015)^{(41)}$ & $\begin{array}{l}\text { Multiple weight (weight, BMI, } \\
\text { WC) }\end{array}$ & $\checkmark$ & & & \\
\hline Yang et al. $(2016)^{(33)}$ & $\begin{array}{l}\text { Multiple weight (weight, BMI, } \\
\text { WC) }\end{array}$ & $\checkmark$ & & & \\
\hline Jebb et al. $(2011)^{(31)}$ & Multiple weight (weight, WC) & $\checkmark$ & & & \\
\hline Debussche et al. $(2018)^{(24)}$ & Multiple weight (BMI, WC) & $\checkmark$ & & & \\
\hline Heshka et al. $(2003)^{(16)}$ & $\begin{array}{l}\text { Multiple weight (weight, BMI, } \\
\text { WC/WHR) }\end{array}$ & $\checkmark$ & & & \\
\hline Corkery et al. (1997) ${ }^{(44)}$ & Dietary pattern & & $\checkmark$ & & \\
\hline Gary et al. $(2003)^{(49)}$ & Dietary pattern & & $\checkmark$ & & \\
\hline Stoddard et al. (2004) & FV & & $\checkmark$ & & \\
\hline Allicock et al. (2012) ${ }^{(67)}$ & $\mathrm{FV}$ & & $\checkmark$ & & \\
\hline Van der Wulp et al. $(2012)^{(51)}$ & Fat & & $\checkmark$ & & \\
\hline Glasgow et al. $(2003)^{(38)}$ & $\begin{array}{l}\text { Multiple dietary (fat, other } \\
\text { nutrients/foods) }\end{array}$ & & $\checkmark$ & & \\
\hline Prezio et al. $(2013)^{(47)}$ & $\mathrm{BMI}$ & & $\checkmark$ & & \\
\hline Peimani et al. $(2018)^{(57)}$ & BMI & & $\checkmark$ & & \\
\hline Islam et al. $(2013)^{(60)}$ & $\begin{array}{l}\text { Multiple weight (weight, BMI, } \\
\text { WC) }\end{array}$ & & $\checkmark$ & & \\
\hline Pérez-Escamilla et al. $(2015)^{(46)}$ & $\begin{array}{l}\text { Multiple weight (weight, BMI, } \\
\text { WC) }\end{array}$ & & $\checkmark$ & & \\
\hline Verheijden et al. (2004) & Multiple weight (BMI, WC/WHR) & & $\checkmark$ & & \\
\hline Sreedevi et al. $(2017)^{(53)}$ & Multiple weight (BMI, WHR) & & $\checkmark$ & & \\
\hline Carrasquillo et al. (2017) ${ }^{(65)}$ & Diet and weight (FV, BMI) & & $\checkmark$ & & \\
\hline Cade et al. $(2009)^{(19)}$ & $\begin{array}{l}\text { Diet and weight (FV, fat, other } \\
\text { nutrients/foods, weight, } \mathrm{BMI})\end{array}$ & & $\checkmark$ & & \\
\hline Spencer et al. $(2011)^{(64)}$ & $\begin{array}{l}\text { Diet and weight (FV, fat, other } \\
\text { nutrients/foods, BMI) }\end{array}$ & & $\checkmark$ & & \\
\hline Mash et al. $(2014)^{(23)}$ & $\begin{array}{l}\text { Diet and weight (dietary pattern, } \\
\text { weight, WC) }\end{array}$ & & $\checkmark$ & & \\
\hline Xiang et al. $(2017)^{(18)}$ & $\begin{array}{l}\text { Diet and weight (dietary pattern, } \\
\text { BMI) }\end{array}$ & & $\checkmark$ & & \\
\hline Toobert et al. (2011) & Fat & & & $\checkmark$ & \\
\hline Perri et al. $(1987)^{(14)}$ & Weight & & & $\checkmark$ & \\
\hline Jolly et al. (2011) ${ }^{(66)}$ & Weight & & & $\checkmark$ & \\
\hline Staten et al. $(2004)^{(62)}$ & $\begin{array}{l}\text { Diet and weight (FV, BMI, WC/ } \\
\text { WHR) }\end{array}$ & & & $\checkmark$ & \\
\hline MacKinnon et al. $(2010)^{(30)}$ & Diet and weight (FV, BMI) & & & $\checkmark$ & \\
\hline Winett et al. $(2007)^{(37)}$ & $\begin{array}{l}\text { Diet and weight (FV, fat, other } \\
\text { nutrients/foods, weight) }\end{array}$ & & & $\checkmark$ & \\
\hline Sternfeld et al. (2009) ${ }^{(40)}$ & $\begin{array}{l}\text { Multiple dietary (FV, fat, other } \\
\text { nutrients/foods) }\end{array}$ & & & & $\checkmark$ \\
\hline Chang et al. $(2017)^{(43)}$ & $\begin{array}{l}\text { Multiple dietary (FV, fat, other } \\
\text { nutrients/foods) }\end{array}$ & & & & $\checkmark$ \\
\hline Djuric et al. $(2002)^{(15)}$ & Multiple weight (weight, BMI) & & & & $\checkmark$ \\
\hline McNabb et al. $(1997)^{(25)}$ & $\begin{array}{l}\text { Diet and weight (fat, other } \\
\text { nutrients/foods, weight, BMI, } \\
\text { WC/WHR) }\end{array}$ & & & & $\checkmark$ \\
\hline Keyserling et al. $(2008)^{(63)}$ & $\begin{array}{l}\text { Diet and weight (dietary pattern, } \\
\text { FV, weight) }\end{array}$ & & & & $\checkmark$ \\
\hline Babamoto et al. (2009) ${ }^{(50)}$ & Diet and weight (FV, fat, BMI) & & & & $\checkmark$ \\
\hline Lorig et al. $(2009)^{(20)}$ & $\begin{array}{l}\text { Diet and weight (dietary pattern, } \\
\text { fat, weight) }\end{array}$ & & & & $\checkmark$ \\
\hline
\end{tabular}




\begin{tabular}{|c|c|c|c|c|c|}
\hline \multirow[b]{2}{*}{ Study } & \multirow[b]{2}{*}{$\begin{array}{l}\text { Dietary behaviour change } \\
\text { outcome(s) measured }\end{array}$} & \multicolumn{4}{|c|}{ Effect of PSI } \\
\hline & & $\begin{array}{l}\text { PSI more } \\
\text { effective than } \\
\text { alternative }\end{array}$ & $\begin{array}{l}\text { No significant } \\
\text { difference between } \\
\text { PSI and control }\end{array}$ & $\begin{array}{l}\text { Mixed within } \\
\text { each } \\
\text { outcome* }\end{array}$ & $\begin{array}{l}\text { Mixed } \\
\text { between } \\
\text { outcomes† }\end{array}$ \\
\hline Balcazar et al. (2010) $)^{(59)}$ & $\begin{array}{l}\text { Diet and weight (fat, other } \\
\text { nutrients/foods, weight, BMI, } \\
\text { WC/WHR) }\end{array}$ & & & & $\checkmark$ \\
\hline Parikh et al. $(2010)^{(21)}$ & $\begin{array}{l}\text { Diet and weight (FV, fat, other } \\
\text { nutrients/foods, weight, WC) }\end{array}$ & & & & $\checkmark$ \\
\hline Rosal et al. (2011) & $\begin{array}{l}\text { Diet and weight (dietary pattern, } \\
\text { fat, other nutrients/foods, } \\
\text { weight, WC) }\end{array}$ & & & & $\checkmark$ \\
\hline Smith et al. (2011) & $\begin{array}{l}\text { Diet and weight (dietary pattern, } \\
\text { BMI) }\end{array}$ & & & & $\checkmark$ \\
\hline Pinto et al. $(2013)^{(17)}$ & $\begin{array}{l}\text { Diet and weight (other nutrients/ } \\
\text { foods, weight) }\end{array}$ & & & & $\checkmark$ \\
\hline Rothschild et al. (2014) $)^{(45)}$ & Diet and weight (FV, weight) & & & & $\checkmark$ \\
\hline Baig et al. (2015) & $\begin{array}{l}\text { Diet and weight (dietary pattern, } \\
\text { FV, fat, WC) }\end{array}$ & & & & $\checkmark$ \\
\hline Hageman et al. $(2017)^{(36)}$ & $\begin{array}{l}\text { Diet and weight (fat, other } \\
\text { nutrients/foods, weight, WC) }\end{array}$ & & & & $\checkmark$ \\
\hline Jane et al. $(2017)^{(42)}$ & $\begin{array}{l}\text { Diet and weight (fat, other } \\
\text { nutrients/foods, weight, BMI, } \\
\text { WC) }\end{array}$ & & & & $\checkmark$ \\
\hline
\end{tabular}

FV, fruit and vegetables; WC, waist circumference; WHR, waist-to-hip ratio.

*Mixed within each outcome: different results for different intervention groups/at different time points.

†Mixed between outcomes: different results for different dietary behaviour change outcome measures.

of obtaining measurements were described in only eighteen ( $45 \%$ ) of these studies. Studies often measured more than one anthropometric outcome, therefore results on the effect of PSI on these measurements are based on a number of the same studies. Of the twenty-seven studies examining the effect of PSI on weight (Table 3), twelve studies (44\%) reported that PSI were more effective than alternative interventions and/or a control for improving weight, ten studies (37\%) reported no significant difference in weight between PSI and alternative interventions and/or a control, and five studies (19\%) reported mixed results. Of the twenty-three studies examining the effect of PSI on BMI, eight studies (35\%) reported PSI were more effective than a control for improving BMI, thirteen studies (57\%) reported no significant difference in BMI between PSI and alternative interventions and/or a control, and two studies (9\%) reported mixed results. Of the eighteen studies examining the effect of PSI on waist and hip measurements, seven studies (39\%) reported that PSI were more effective than a control for improving waist and hip measurements; eleven studies (61\%) reported no significant difference in waist and hip measurements between PSI and alternative interventions and/or a control. Studies measuring anthropometric outcomes that found positive effects of PSI were largely weight management interventions, whereas studies reporting no significant difference between PSI and alternative interventions and/or a control or mixed results were generally behaviour change interventions that include improving diet rather than explicit weight loss.

\section{Effect of peer-supported intervention characteristics on dietary behaviour change}

As demonstrated in Table 4, included studies were published from 1987 to 2018. Studies compared a PSI with a control ( $n 42 ; 78 \%$ ) or an alternative support intervention and control ( $n$ 12; $22 \%$ ). Key elements of PSI included: education, use of BCT and provision of emotional support. The total number of participants included was 18144, of whom 8115 received a PSI. Numbers receiving the PSI in studies ranged from $<10$ to $>1000$. Most studies included both genders ( $n 42 ; 78 \%$ ), but approximately one-quarter of studies included females only ( $n 12 ; 22 \%$ ). The mean age of participants reported ranged from 28 to 71 years; where only an age range was reported, the youngest age was 21 years and the oldest was 70 years.

This section of the results presents PSI characteristics that were analysed to determine if they were linked with effectiveness in encouraging dietary behaviour change, which included PSI model used, peer role, peer attributes, behaviour change theory/techniques used, intervention duration and intensity, and population studied (ethnicity and characteristics). Findings on peer role and attributes also include a description of peer training and support and measurement of fidelity of PSI delivery across studies.

PSI were highly heterogeneous. PSI models used included group ( $n$ 30; 56\%), dyadic ( $n$ 10; 19\%) and a hybrid of models ( $n$ 14; $26 \%$ ). Group support was largely delivered face-to-face ( $n 22 ; 73 \%$ ) or was delivered via teleconference ( $n 1 ; 3 \%)$ or was web-based $(n 7 ; 23 \%)$. Dyadic support was delivered face-to-face ( $n$ 4; $40 \%$ ), via 
Table 2 Effect of peer-supported interventions (PSI) on dietary outcomes ( $n$ 35)

\begin{tabular}{|c|c|c|c|c|}
\hline \multirow[b]{2}{*}{ Study } & \multirow[b]{2}{*}{ Outcome measure } & \multirow[b]{2}{*}{ Intervention groups } & \multicolumn{2}{|c|}{$\begin{array}{l}\text { Effect of } \\
\text { PSI }^{*}\end{array}$} \\
\hline & & & $\uparrow c$ & C $\quad 1 \quad \downarrow$ \\
\hline \multicolumn{5}{|l|}{ Dietary pattern $(n 11)$} \\
\hline Garrett et al. (2005) $^{(28)}$ & Six-item q'naire on diabetes self-care & $\begin{array}{l}\text { 1. Small group activity (PSI) } \\
\text { 2. Control }\end{array}$ & $\checkmark$ & \\
\hline $\begin{array}{l}\text { Robinson-Whelen et al. } \\
(2006)^{(32)}\end{array}$ & $\begin{array}{l}\text { Forty-eight-item q'naire on health } \\
\text { behaviours }\end{array}$ & $\begin{array}{l}\text { 1. Health promotion programme (PSI) } \\
\text { 2. Control }\end{array}$ & $\checkmark$ & \\
\hline Keyserling et al. (2008) ${ }^{63)}$ & $\begin{array}{l}\text { Fifty-four-item FFQ (v) (New Leaf Dietary } \\
\text { Risk Assessment) }\end{array}$ & $\begin{array}{l}\text { 1. Enhanced intervention (PSI) } \\
\text { 2. Minimal intervention }\end{array}$ & $\checkmark$ & \\
\hline Lorig et al. (2009) & $\begin{array}{l}\text { Three-item FFQ to measure healthy } \\
\text { eating practices }\end{array}$ & $\begin{array}{l}\text { 1. Peer-led diabetes self-management programme } \\
\text { (PSI) } \\
\text { 2. Usual care }\end{array}$ & $\checkmark$ & \\
\hline Rosal et al. (2011) & $\begin{array}{l}24 \mathrm{~h} \text { dietary recalls }(\times 3 \text { at baseline, } \times 1 \text { at } \\
4 \text { months, } \times 3 \text { at } 12 \text { months) }\end{array}$ & $\begin{array}{l}\text { 1. Tailored diabetes self-management } \\
\text { 2. Usual care }\end{array}$ & $\checkmark$ & \\
\hline Corkery et al. $(1997)^{(44)}$ & Ten-item q'naire on diabetes self-care & $\begin{array}{l}\text { 1. PSI } \\
\text { 2. Control }\end{array}$ & & $\checkmark$ \\
\hline Gary et al. $(2003)^{(49)}$ & FFQ (v) & $\begin{array}{l}\text { 1. Usual care } \\
\text { 2. Usual care and PSI } \\
\text { 3. Usual care + nurse case manager } \\
\text { 4. Usual care + nurse case manager + PSI }\end{array}$ & & $\checkmark$ \\
\hline Smith et al. $(2011)^{(22)}$ & Diabetes self-care self-report q'naire $(v)$ & $\begin{array}{l}\text { 1. PSI } \\
\text { 2. Control }\end{array}$ & & $\checkmark$ \\
\hline Mash et al. $(2014)^{(23)}$ & $\begin{array}{l}\text { One item from Summary of Diabetes } \\
\text { Self-Care Activity Scale }\end{array}$ & $\begin{array}{l}\text { 1. Diabetes education programme } \\
\text { 2. Usual care and education }\end{array}$ & & $\checkmark$ \\
\hline Baig et al. $(2015)^{(27)}$ & $\begin{array}{l}\text { One item from Summary of Diabetes } \\
\text { Self-Care Activity Scale }\end{array}$ & $\begin{array}{l}\text { 1. Church-based self-management intervention } \\
\text { 2. Usual care and education }\end{array}$ & & $\checkmark$ \\
\hline Xiang et al. $(2017)^{(18)}$ & $\begin{array}{l}\text { Diabetes self-care self-report q'naire } \\
\text { including four items on diet (v) }\end{array}$ & $\begin{array}{l}\text { 1. Patient-to-patient education } \\
\text { 2. Control }\end{array}$ & & $\checkmark$ \\
\hline \multicolumn{5}{|l|}{ FV intake $(n 17)$} \\
\hline Campbell et al. $(1999)^{(55)}$ & $\begin{array}{l}\text { Fifteen-item FFQ (validated against } 3 d \\
\text { food records) }(v)\end{array}$ & $\begin{array}{l}\text { 1. Five-a-day intervention (PSI) } \\
\text { 2. Control }\end{array}$ & $\checkmark$ & \\
\hline Resnicow et al. $(2004)^{(58)}$ & $\begin{array}{l}\text { Seventeen-item FV FFQ and two-item } \\
\text { q'naire of usual FV intake/d (v) }\end{array}$ & $\begin{array}{l}\text { 1. Body and Soul intervention (PSI) } \\
\text { 2. Control }\end{array}$ & $\checkmark$ & \\
\hline Keyserling et al. $(2008)^{63)}$ & Serum carotenoids & $\begin{array}{l}\text { 1. Enhanced intervention (PSI) } \\
\text { 2. Minimal intervention }\end{array}$ & $\checkmark$ & \\
\hline $\begin{array}{l}\text { Babamoto et al. } \\
\quad(2009)^{(50)}\end{array}$ & Diabetes health measures q'naire & $\begin{array}{l}\text { 1. CHW education (PSI) } \\
\text { 2. Case management } \\
\text { 3. Usual care }\end{array}$ & $\checkmark$ & \\
\hline Sternfeld et al. $(2009)^{(40)}$ & FFQ (v) & $\begin{array}{l}\text { 1. A Lifestyle Intervention Via Email (ALIVE) } \\
\text { programme (PSI) } \\
\text { 2. Control }\end{array}$ & $\checkmark$ & \\
\hline Winett et al. $(2007)^{(37)} \dagger$ & $\mathrm{FFQ}(\mathrm{v})$ & $\begin{array}{l}\text { 1. Guide to Health intervention (GTH) } \\
\text { 2. GTH with church-based supports (PSI) } \\
\text { 3. Control }\end{array}$ & $\checkmark$ & $\checkmark$ \\
\hline $\begin{array}{l}\text { Mackinnon et al. } \\
\quad(2010)^{(30)} \ddagger\end{array}$ & Dietary q'naire & $\begin{array}{l}\text { 1. Team-based, peer-led scripted health promotion } \\
\text { (PSI) } \\
\text { 2. Control }\end{array}$ & $\checkmark \checkmark$ & \\
\hline Stoddard et al. $(2004)^{(56)}$ & $\begin{array}{l}\text { One-item assessment on average } \\
\text { servings of } \mathrm{FV} / \mathrm{d}\end{array}$ & $\begin{array}{l}\text { 1. Enhanced intervention (PSI) } \\
\text { 2. Minimal intervention }\end{array}$ & & $\checkmark$ \\
\hline Cade et al. $(2009)^{(19)}$ & $3 \mathrm{~d}$ food diary and q'naire $(\mathrm{v})$ & $\begin{array}{l}\text { 1. Diabetes-specific expert patient programme (PSI) } \\
\text { 2. Individual dietetic counselling control }\end{array}$ & & $\checkmark$ \\
\hline Parikh et al. (2010) & FFQ and self-report dietary q'naire (v) & $\begin{array}{l}\text { 1. Peer-led lifestyle intervention } \\
\text { 2. Delayed intervention control }\end{array}$ & & $\checkmark$ \\
\hline Spencer et al. $(2011)^{(64)}$ & $\begin{array}{l}\text { Six-item interview-administered FFQ } \\
\text { from CDC Behavioral Risk Factor } \\
\text { Surveillance System (v) }\end{array}$ & $\begin{array}{l}\text { 1. Diabetes self-management } \\
\text { 2. Delayed control }\end{array}$ & & $\checkmark$ \\
\hline Allicock et al. $(2012)^{(67)}$ & $\begin{array}{l}\text { Nine-item FV q'naire and two-item } \\
\text { measure of usual FV intake (v) }\end{array}$ & $\begin{array}{l}\text { 1. Body and Soul group (PSI) } \\
\text { 2. Control }\end{array}$ & & $\checkmark$ \\
\hline $\begin{array}{l}\text { Rothschild et al. } \\
\qquad(2014)^{(45)}\end{array}$ & $\begin{array}{l}\text { One item from Summary of Diabetes } \\
\text { Self-Care Activity Scale }\end{array}$ & $\begin{array}{l}\text { 1. Self-management intervention } \\
\text { 2. Education-only control }\end{array}$ & & $\checkmark$ \\
\hline Baig et al. $(2015)^{(27)}$ & $\begin{array}{l}\text { One item from Summary of Diabetes } \\
\text { Self-Care Activity Scale }\end{array}$ & $\begin{array}{l}\text { 1. Church-based self-management intervention } \\
\text { 2. Usual care and education }\end{array}$ & & $\checkmark$ \\
\hline $\begin{array}{l}\text { Carrasquillo et al. } \\
(2017)^{(65)}\end{array}$ & Six-item interview-administered FFQ (v) & $\begin{array}{l}\text { 1. CHW intervention } \\
\text { 2. Enhanced usual care }\end{array}$ & & $\checkmark$ \\
\hline Chang et al. $(2017)^{(43)}$ & Seven-item FV FFQ (v) & $\begin{array}{l}\text { 1. PSI } \\
\text { 2. Written information control }\end{array}$ & & $\checkmark$ \\
\hline Staten et al. $(2004)^{(62)}$ & $24 \mathrm{~h}$ diet recall & $\begin{array}{l}\text { 1. Counselling - active control } \\
\text { 2. Counselling and health education } \\
\text { 3. Counselling, health education and PSI }\end{array}$ & & $\checkmark \checkmark$ \\
\hline
\end{tabular}




\begin{tabular}{|c|c|c|c|c|}
\hline \multirow[b]{2}{*}{ Study } & \multirow[b]{2}{*}{ Outcome measure } & \multirow[b]{2}{*}{ Intervention groups } & \multicolumn{2}{|c|}{$\begin{array}{l}\text { Effect of } \\
\text { PSI }^{*}\end{array}$} \\
\hline & & & $\uparrow \mathrm{C}$ & C $\quad I \quad \downarrow$ \\
\hline \multicolumn{5}{|l|}{ Fat intake ( $n$ 18) } \\
\hline McNabb et al. $(1997)^{(25)}$ & $\begin{array}{l}\text { Dietary recall checklist on fat and fibre } \\
\text { intake }(v)\end{array}$ & $\begin{array}{l}\text { 1. Experimental group (PSI) } \\
\text { 2. Wait list control }\end{array}$ & \multicolumn{2}{|l|}{$\checkmark$} \\
\hline Resnicow et al. $(2004)^{(58)}$ & Fifteen-item q'naire on fat intake (v) & $\begin{array}{l}\text { 1. Body and Soul intervention (PSI) } \\
\text { 2. Control }\end{array}$ & \multicolumn{2}{|l|}{$\checkmark$} \\
\hline Toobert et al. $(2007)^{(35)}$ & $\begin{array}{l}\text { FFQ to document \% energy from } \\
\text { saturated fat. Validated with plasma } \\
\text { fatty acids }(\mathrm{v})\end{array}$ & $\begin{array}{l}\text { 1. Mediterranean lifestyle programme (PSI) } \\
\text { 2. Usual care }\end{array}$ & \multicolumn{2}{|l|}{$\checkmark$} \\
\hline Sternfeld et al. (2009) $)^{(40)}$ & FFQ $(v)$ & $\begin{array}{l}\text { 1. A Lifestyle Intervention Via Email (ALIVE) } \\
\text { programme (PSI) } \\
\text { 2. Control }\end{array}$ & \multicolumn{2}{|l|}{$\checkmark$} \\
\hline Balcazar et al. $(2010)^{(59)}$ & Thirty-five-item behavioural habits q'naire & $\begin{array}{l}\text { 1. CHW education (PSI) } \\
\text { 2. Control }\end{array}$ & \multicolumn{2}{|l|}{$\checkmark$} \\
\hline Rosal et al. $(2011)^{(54)}$ & $\begin{array}{l}24 \text { h dietary recalls ( } \times 3 \text { at baseline, } \times 1 \text { at } \\
4 \text { months, } \times 3 \text { at } 12 \text { months) }\end{array}$ & $\begin{array}{l}\text { 1. Tailored diabetes self-management } \\
\text { 2. Usual care }\end{array}$ & \multicolumn{2}{|l|}{$\checkmark$} \\
\hline Baig et al. $(2015)^{(27)}$ & $\begin{array}{l}\text { One item from Summary of Diabetes } \\
\text { Self-Care Activity Scale }\end{array}$ & $\begin{array}{l}\text { 1. Church-based self-management intervention } \\
\text { 2. Usual care and education }\end{array}$ & \multicolumn{2}{|l|}{$\checkmark$} \\
\hline Chang et al. $(2017)^{(43)}$ & Seventeen-item FFQ (v) & $\begin{array}{l}\text { 1. PSI } \\
\text { 2. Written information control }\end{array}$ & \multicolumn{2}{|l|}{$\checkmark$} \\
\hline Toobert et al. $(2011)^{(34)} \ddagger$ & FFQ to document \% energy from fat $(v)$ & $\begin{array}{l}\text { 1. Viva Bien! and usual care (PSI) } \\
\text { 2. Usual care }\end{array}$ & \multicolumn{2}{|c|}{$\checkmark \checkmark$} \\
\hline Glasgow et al. (2003) $)^{(38)}$ & $\begin{array}{l}\text { Fifteen-item q'naire on fat intake and } \\
\text { twenty-item fat \& fibre behaviour } \\
\text { q'naire }(v)\end{array}$ & $\begin{array}{l}\text { 1. Information only } \\
\text { 2. PSI }\end{array}$ & \multicolumn{2}{|c|}{$\checkmark$} \\
\hline Cade et al. $(2009)^{(19)}$ & $3 \mathrm{~d}$ food diary and q'naire $(\mathrm{v})$ & $\begin{array}{l}\text { 1. Diabetes-specific expert patient programme (PSI) } \\
\text { 2. Individual dietetic counselling control }\end{array}$ & \multicolumn{2}{|c|}{$\checkmark$} \\
\hline Parikh et al. (2010) & FFQ and self-report dietary q'naire (v) & $\begin{array}{l}\text { 1. Peer-led lifestyle intervention } \\
\text { 2. Delayed intervention control }\end{array}$ & \multicolumn{2}{|c|}{$\checkmark$} \\
\hline Spencer et al. $(2011)^{(64)}$ & $\begin{array}{l}\text { Thirteen-item interview-administered } \\
\text { FFQ from CDC Behavioral Risk Factor } \\
\text { Surveillance System (v) }\end{array}$ & $\begin{array}{l}\text { 1. Diabetes self-management } \\
\text { 2. Delayed control }\end{array}$ & \multicolumn{2}{|c|}{$\checkmark$} \\
\hline $\begin{array}{l}\text { Van der Wulp et al. } \\
(2012)^{(51)}\end{array}$ & $\begin{array}{l}\text { Thirty-five-item q'naire on fat intake } \\
\text { (Fatlist) }\end{array}$ & $\begin{array}{l}\text { 1. Peer-led self-management (PSI) } \\
\text { 2. Control }\end{array}$ & \multicolumn{2}{|c|}{$\checkmark$} \\
\hline Winett et al. $(2007)^{(37)}$ & FFQ to document \% energy from fat $(v)$ & $\begin{array}{l}\text { 1. Guide to Health intervention (GTH) } \\
\text { 2. GTH with church-based supports (PSI) } \\
\text { 3. Control }\end{array}$ & \multicolumn{2}{|c|}{$\checkmark \checkmark$} \\
\hline $\begin{array}{l}\text { Babamoto et al. } \\
\qquad(2009)^{(50)}\end{array}$ & Diabetes health measures q'naire & $\begin{array}{l}\text { 1. CHW education (PSI) } \\
\text { 2. Case management } \\
\text { 3. Usual care }\end{array}$ & \multicolumn{2}{|c|}{$\checkmark \sqrt{ }$} \\
\hline Jane et al. $(2017)^{(42)}$ & $3 \mathrm{~d}$ food record & $\begin{array}{l}\text { 1. Social media-delivered weight management } \\
\text { programme } \\
\text { 2. Written information } \\
\text { 3. Standard care }\end{array}$ & \multicolumn{2}{|c|}{$\checkmark \checkmark$} \\
\hline $\begin{array}{l}\text { Hageman et al. } \\
\qquad(2017)^{(36)} \ddagger\end{array}$ & $\begin{array}{l}\text { Block Health Habit and History } \\
\text { Questionnaire (v) }\end{array}$ & $\begin{array}{l}\text { 1. Web intervention } \\
\text { 2. Web intervention + PSI } \\
\text { 3. Web intervention + professional counselling }\end{array}$ & \multicolumn{2}{|c|}{ 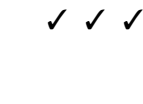 } \\
\hline \multicolumn{5}{|c|}{ Intake of other nutrients/foods ( $n$ 13) } \\
\hline Balcazar et al. $(2010)^{(59)}$ & $\begin{array}{l}\text { Salt: } \\
\text { Thirty-five-item behavioural habits } \\
\text { q'naire }\end{array}$ & $\begin{array}{l}\text { 1. CHW education (PSI) } \\
\text { 2. Control }\end{array}$ & \multicolumn{2}{|l|}{$\checkmark$} \\
\hline Winett et al. $(2007)^{(37)} \dagger$ & $\begin{array}{l}\text { Fibre: } \\
\text { FFQ to document } \% \text { energy from fibre } \\
\text { (v) servings }\end{array}$ & $\begin{array}{l}\text { 1. Guide to Health intervention (GTH) } \\
\text { 2. GTH with church-based supports (PSI) } \\
\text { 3. Control }\end{array}$ & \multicolumn{2}{|l|}{$\checkmark$} \\
\hline McNabb et al. $(1997)^{(25)}$ & $\begin{array}{l}\text { Fibre: } \\
\text { Dietary recall checklist on fat and fibre } \\
\text { intake }(v)\end{array}$ & $\begin{array}{l}\text { 1. Experimental group (PSI) } \\
\text { 2. Wait list control }\end{array}$ & \multicolumn{2}{|c|}{$\checkmark$} \\
\hline Glasgow et al. (2003) $)^{(38)}$ & $\begin{array}{l}\text { Fibre: } \\
\text { Twenty-item fat \& fibre behaviour q'naire } \\
\text { (v) }\end{array}$ & $\begin{array}{l}\text { 1. Information only } \\
\text { 2. PSI }\end{array}$ & \multicolumn{2}{|c|}{$\checkmark$} \\
\hline Cade et al. (2009) $)^{(19)}$ & $\begin{array}{l}\text { Energy, protein, starch, fibre: } \\
3 \text { d food diary and q'naire }\end{array}$ & $\begin{array}{l}\text { 1. Diabetes-specific expert patient programme (PSI) } \\
\text { 2. Individual dietetic counselling control }\end{array}$ & \multicolumn{2}{|c|}{$\checkmark$} \\
\hline Sternfeld et al. (2009) ${ }^{(40)}$ & $\begin{array}{l}\text { Sugar: } \\
\text { FFQ (v) }\end{array}$ & $\begin{array}{l}\text { 1. A Lifestyle Intervention Via Email (ALIVE) } \\
\text { programme (PSI) } \\
\text { 2. Control }\end{array}$ & \multicolumn{2}{|c|}{$\checkmark$} \\
\hline Parikh et al. (2010) & $\begin{array}{l}\text { Sugar-sweetened beverages: } \\
\text { FFQ and self-report dietary q'naire (v) }\end{array}$ & $\begin{array}{l}\text { 1. Peer-led lifestyle intervention } \\
\text { 2. Delayed intervention control }\end{array}$ & \multicolumn{2}{|c|}{$\checkmark$} \\
\hline
\end{tabular}




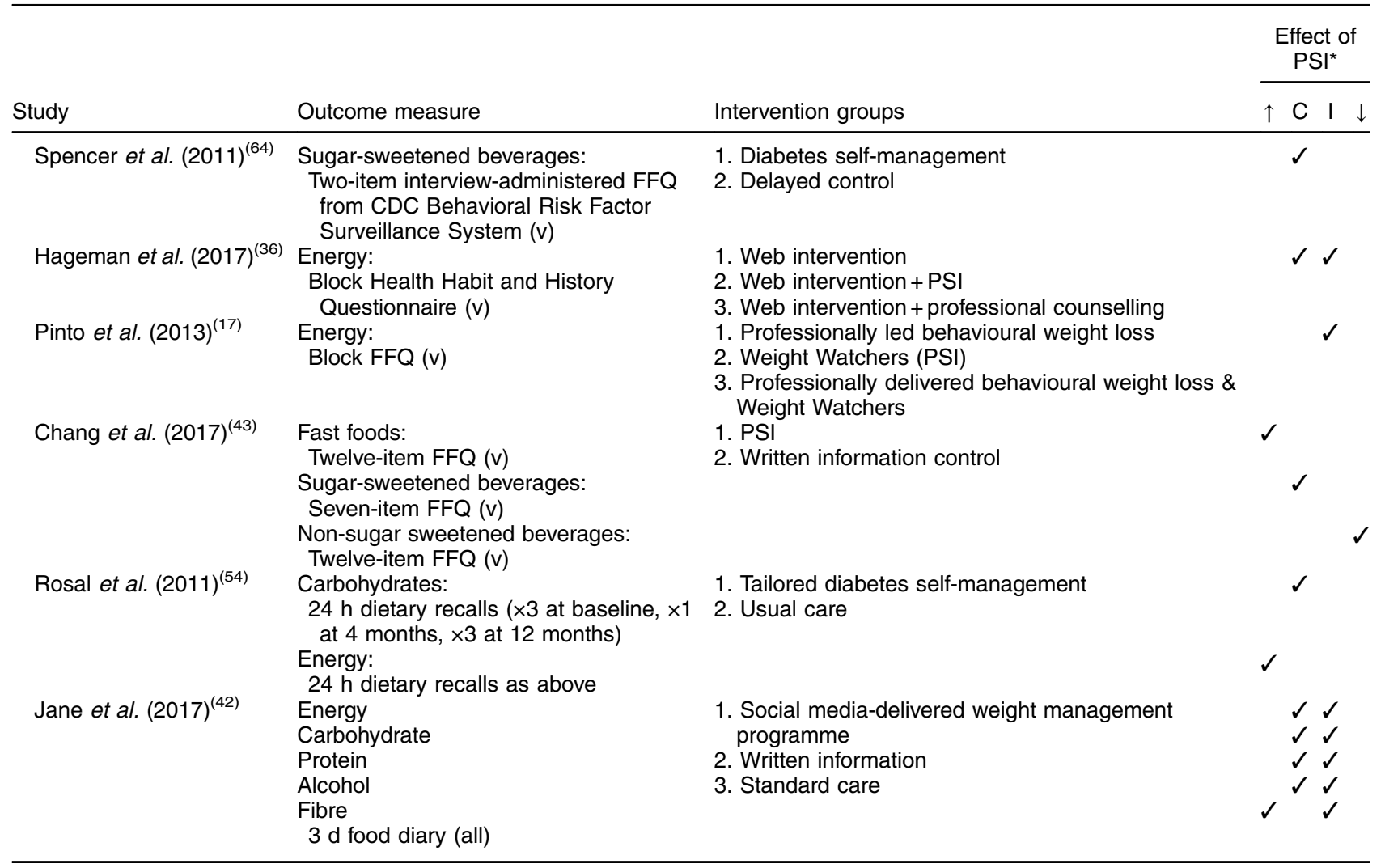

FV, fruit and vegetable; q,naire, questionnaire; v, validated; CDC, Centers for disease control and Prevention; CHW, Community Health Worker. ${ }^{*}$ Effect of PSI summarised as: $\uparrow$, PSI more effective than alternative method of support in improving dietary outcome; C, no significant difference between PSI and control group in improving dietary outcome; I, no significant difference between PSI and intervention group in improving dietary outcome; $\downarrow$, PSI less effective than other method of support in improving dietary outcome.

†Different results for different intervention groups.

$\ddagger$ Different results at different time points.

telephone $(n 1 ; 10 \%)$ or via face-to-face and/or telephone ( $n$ 5; 50\%). There were no clear differences in PSI model between the fifteen studies that reported PSI to be a more effective form of support $v$, the studies that reported no significant difference between support methods. Of studies using a group PSI ( $n$ 30), approximately one-third reported positive effects of PSI ( $n$ 10; 33\%); whereas of studies using a dyadic PSI ( $n$ 10), one-fifth reported positive effects of PSI ( $n 2 ; 20 \%$ ) and of studies using a hybrid PSI ( $n$ 14), approximately one-fifth reported positive effects of PSI ( $n$ 3; $21 \%$ ). Of studies measuring the effect of PSI on dietary outcomes, there was no clear difference in PSI models used in studies that reported PSI to be a more effective form of support and studies reporting no significant difference between methods of support. Studies that reported PSI to be more effective for improving weight, BMI and waist measurements mostly used group-based support, whereas studies that reported no significant difference in methods of support for anthropometric outcomes used a range of PSI models.

In six studies, the PSI was delivered by study participants to one another. In the remaining forty-eight studies, the PSI was peer-led by lay individuals ( $n$ 30; 63\%), CHW
( $n$ 9; 19\%), CHW and health professionals ( $n$ 4; $8 \%$ ), and lay individuals and health professionals ( $n$; $10 \%)$. PSI were delivered via a commercial programme in a small number of studies $(n 6 ; 11 \%)$. Peer roles included a peer supporter ( $n$ 24), peer educator ( $n$ 22), peer counsellor ( $n$ 22 ), peer facilitator $(n 6)$ and peer case manager $(n 5)$. Studies that reported PSI to be a more effective form of support ( $n$ 15; 28\%) mostly used lay-led support ( $n$ 11; $73 \%)$, whereas studies reporting no significant difference between methods of support ( $n 17 ; 31 \%$ ) used a range of individuals to deliver the PSI including lay individuals ( $n$ 8; $47 \%$ ), other participants ( $n 3 ; 18 \%) \mathrm{CHW}(n 5 ; 29 \%)$ and a combination of CHW and health professionals ( $n 1 ; 6 \%$ ). There were no clear differences in peer role between the studies that reported PSI to be a more effective form of support $v$. the studies that reported no significant difference between support methods. Studies where the peer assumed the role of peer case manager ( $n 2 ; 4 \%$ ) both reported no significant difference, whereas other peer roles were used in studies reporting PSI to be a more effective form of support and studies reporting no significant difference between support methods. Over half of studies that reported positive effects of PSI ( $n$ 15; 28\%) 
Table 3 Effect of peer-supported interventions (PSI) on anthropometric outcomes ( $n$ 40)

\begin{tabular}{|c|c|c|c|c|c|}
\hline \multirow[b]{2}{*}{ Study } & \multirow[b]{2}{*}{ Intervention groups } & \multicolumn{4}{|c|}{ Effect of $\mathrm{PSI}^{*}$} \\
\hline & & $\uparrow$ & $\mathrm{C}$ & $\mathrm{I}$ & $\downarrow$ \\
\hline \multicolumn{6}{|l|}{ Weight ( $n$ 27) } \\
\hline McNabb et al. $(1997)^{(25)}$ & $\begin{array}{l}\text { 1. Experimental group (PSI) } \\
\text { 2. Wait list control }\end{array}$ & $\checkmark$ & & & \\
\hline Heshka et al. $(2003)^{(16)}$ & $\begin{array}{l}\text { 1. Self-help weight loss group } \\
\text { 2. Weight Watchers (PSI) }\end{array}$ & $\checkmark$ & & & \\
\hline Parikh et al. $(2010)^{(21)}$ & $\begin{array}{l}\text { 1. Peer-led lifestyle intervention } \\
\text { 2. Delayed intervention control }\end{array}$ & $\checkmark$ & & & \\
\hline Jebb et al. $(2011)^{(31)}$ & $\begin{array}{l}\text { 1. Weight Watchers (PSI) } \\
\text { 2. Standard care }\end{array}$ & $\checkmark$ & & & \\
\hline West et al. $(2011)^{(26)}$ & $\begin{array}{l}\text { 1. Diabetes prevention programme } \\
\text { 2. Cognitive training }\end{array}$ & $\checkmark$ & & & \\
\hline Katula et al. $(2013)^{(61)}$ & $\begin{array}{l}\text { 1. Diabetes Prevention Program lifestyle weight loss intervention } \\
\text { 2. Enhanced usual care }\end{array}$ & $\checkmark$ & & & \\
\hline Pinto et al. $(2013)^{(17)}$ & $\begin{array}{l}\text { 1. Professionally led behavioural weight loss } \\
\text { 2. Weight Watchers (PSI) } \\
\text { 3. Professionally delivered behavioural weight loss \& Weight Watchers }\end{array}$ & $\checkmark$ & & & \\
\hline Rothschild et al. (2014) ${ }^{(45)}$ & $\begin{array}{l}\text { 1. Self-management intervention } \\
\text { 2. Education-only control }\end{array}$ & $\checkmark$ & & & \\
\hline Ackermann et al. $(2015)^{(29)}$ & $\begin{array}{l}\text { 1. YMCA intervention - group-based counselling } \\
\text { 2. Usual care }\end{array}$ & $\checkmark$ & & & \\
\hline Block et al. (2015) ${ }^{(41)}$ & $\begin{array}{l}\text { 1. Alive-PD intervention } \\
\text { 2. Usual care }\end{array}$ & $\checkmark$ & & & \\
\hline Yang et al. (2016) ${ }^{(33)}$ & $\begin{array}{l}\text { 1. Weight Watchers (PSI) } \\
\text { 2. Nutrition education control }\end{array}$ & $\checkmark$ & & & \\
\hline Lutes et al. $(2017)^{(48)}$ & $\begin{array}{l}\text { 1. Small Changes lifestyle intervention } \\
\text { 2. Mail-based education control }\end{array}$ & $\checkmark$ & & & \\
\hline Jolly et al. $(2011)^{(66)} \dagger$ & $\begin{array}{l}\text { 1. Weight Watchers (PSI) } \\
\text { 2. Slimming World (PSI) } \\
\text { 3. Exercise minimal intervention }\end{array}$ & $\checkmark$ & $\checkmark$ & & \\
\hline Jane et al. $(2017)^{(42)}$ & $\begin{array}{l}\text { 1. Social media-delivered weight management programme } \\
\text { 2. Written information } \\
\text { 3. Standard care }\end{array}$ & $\checkmark$ & & $\checkmark$ & \\
\hline Winett et al. $(2007)^{(37)} \ddagger$ & $\begin{array}{l}\text { 1. Guide to Health intervention (GTH) } \\
\text { 2. GTH with church-based support (PSI) } \\
\text { 3. Control }\end{array}$ & $\checkmark$ & $\checkmark$ & $\checkmark$ & \\
\hline Keyserling et al. (2008) ${ }^{(63)}$ & $\begin{array}{l}\text { 1. Enhanced intervention (PSI) } \\
\text { 2. Minimal intervention }\end{array}$ & & $\checkmark$ & & \\
\hline Cade et al. (2009) ${ }^{(19)}$ & $\begin{array}{l}\text { 1. Diabetes specific expert patient programme (PSI) } \\
\text { 2. Individual dietetic counselling control }\end{array}$ & & $\checkmark$ & & \\
\hline Lorig et al. (2009) ${ }^{(20)}$ & $\begin{array}{l}\text { 1. Peer-led diabetes self-management programme (PSI) } \\
\text { 2. Usual care }\end{array}$ & & $\checkmark$ & & \\
\hline Balcazar et al. $(2010)^{(59)}$ & $\begin{array}{l}\text { 1. CHW education (PSI) } \\
\text { 2. Control }\end{array}$ & & $\checkmark$ & & \\
\hline Rosal et al. $(2011)^{(54)}$ & $\begin{array}{l}\text { 1. Tailored diabetes self-management } \\
\text { 2. Usual care }\end{array}$ & & $\checkmark$ & & \\
\hline Mash et al. (2014) & $\begin{array}{l}\text { 1. Diabetes education programme } \\
\text { 2. Usual care and education }\end{array}$ & & $\checkmark$ & & \\
\hline Islam et al. $(2013)^{(60)}$ & $\begin{array}{l}\text { 1. Project RICE - CHW intervention } \\
\text { 2. Control }\end{array}$ & & $\checkmark$ & & \\
\hline Baig et al. (2015) & $\begin{array}{l}\text { 1. Church-based self-management } \\
\text { 2. Usual care and education }\end{array}$ & & $\checkmark$ & & \\
\hline Pérez-Escamilla et al. $(2015)^{(46)}$ & $\begin{array}{l}\text { 1. Structured blood glucose control programme } \\
\text { 2. Usual care }\end{array}$ & & $\checkmark$ & & \\
\hline Hageman et al. $(2017)^{(36)}$ & $\begin{array}{l}\text { 1. Web intervention } \\
\text { 2. Web intervention + PSI } \\
\text { 3. Web intervention + professional counselling }\end{array}$ & & $\checkmark$ & $\checkmark$ & \\
\hline Perri et al. $(1987)^{(14)} \ddagger$ & $\begin{array}{l}\text { 1. Behaviour therapy + PSI } \\
\text { 2. Behaviour therapy + therapist } \\
\text { 3. Behaviour therapy }\end{array}$ & & $\checkmark$ & $\checkmark$ & $\checkmark$ \\
\hline Djuric et al. $(2002)^{(15)} \dagger$ & $\begin{array}{l}\text { 1. Control } \\
\text { 2. Weight Watchers (PSI) } \\
\text { 3. Individualised counselling } \\
\text { 4. Individualised counselling \& Weight Watchers }\end{array}$ & & $\checkmark$ & & $\checkmark$ \\
\hline \multicolumn{6}{|c|}{ (5) } \\
\hline McNabb et al. $(1997)^{(25)}$ & $\begin{array}{l}\text { 1. Experimental group (PSI) } \\
\text { 2. Wait list control }\end{array}$ & $\checkmark$ & & & \\
\hline Heshka et al. $(2003)^{(16)}$ & $\begin{array}{l}\text { 1. Self-help weight loss group } \\
\text { 2. Weight Watchers (PSI) }\end{array}$ & $\checkmark$ & & & \\
\hline
\end{tabular}


Table 3 Continued

\begin{tabular}{|c|c|c|c|c|c|}
\hline \multirow[b]{2}{*}{ Study } & \multirow[b]{2}{*}{ Intervention groups } & \multicolumn{4}{|c|}{ Effect of PSI* } \\
\hline & & $\uparrow$ & C & 1 & $\downarrow$ \\
\hline Smith et al. (2011) & $\begin{array}{l}\text { 1. PSI } \\
\text { 2. Control }\end{array}$ & $\checkmark$ & & & \\
\hline Katula et al. $(2013)^{(61)}$ & $\begin{array}{l}\text { 1. Diabetes Prevention Program lifestyle weight loss intervention } \\
\text { 2. Enhanced usual care }\end{array}$ & $\checkmark$ & & & \\
\hline Block et al. $(2015)^{(41)}$ & $\begin{array}{l}\text { 1. Alive-PD intervention } \\
\text { 2. Usual care }\end{array}$ & $\checkmark$ & & & \\
\hline Safford et al. (2015) ${ }^{(52)}$ & $\begin{array}{l}\text { 1. PSI and brief education } \\
\text { 2. Brief education }\end{array}$ & $\checkmark$ & & & \\
\hline Yang et al. $(2016)^{(33)}$ & $\begin{array}{l}\text { 1. Weight Watchers (PSI) } \\
\text { 2. Nutrition education control }\end{array}$ & $\checkmark$ & & & \\
\hline Debussche et al. (2018) & $\begin{array}{l}\text { 1. Peer-led self-management education } \\
\text { 2. Usual care }\end{array}$ & $\checkmark$ & & & \\
\hline MacKinnon et al. $(2010)^{(30)} \ddagger$ & $\begin{array}{l}\text { 1. Team-based, peer-led scripted health promotion (PSI) } \\
\text { 2. Control }\end{array}$ & $\checkmark$ & $\checkmark$ & & \\
\hline Babamoto et al. $(2009)^{(50)} \dagger$ & $\begin{array}{l}\text { 1. CHW education (PSI) } \\
\text { 2. Case management } \\
\text { 3. Usual care }\end{array}$ & $\checkmark$ & & $\checkmark$ & \\
\hline Djuric et al. $(2002)^{(15)}$ & $\begin{array}{l}\text { 1. Control } \\
\text { 2. Weight Watchers (PSI) } \\
\text { 3. Individualised counselling } \\
\text { 4. Individualised counselling \& Weight Watchers }\end{array}$ & & $\checkmark$ & & \\
\hline Verheijden et al. (2004) ${ }^{(39)}$ & $\begin{array}{l}\text { 1. Web-based intervention } \\
\text { 2. Control }\end{array}$ & & $\checkmark$ & & \\
\hline Cade et al. (2009) $)^{(19)}$ & $\begin{array}{l}\text { 1. Diabetes-specific expert patient programme (PSI) } \\
\text { 2. Individual dietetic counselling control }\end{array}$ & & $\checkmark$ & & \\
\hline Balcazar et al. (2010) ${ }^{(59)}$ & $\begin{array}{l}\text { 1. CHW education (PSI) } \\
\text { 2. Control }\end{array}$ & & $\checkmark$ & & \\
\hline Spencer et al. $(2011)^{(64)}$ & $\begin{array}{l}\text { 1. Diabetes self-management } \\
\text { 2. Delayed control }\end{array}$ & & $\checkmark$ & & \\
\hline Islam et al. $(2013)^{(60)}$ & $\begin{array}{l}\text { 1. Project RICE - CHW intervention } \\
\text { 2. Control }\end{array}$ & & $\checkmark$ & & \\
\hline Prezio et al. $(2013)^{(47)}$ & $\begin{array}{l}\text { 1. Culturally tailored diabetes education and management programme } \\
\text { 2. Usual care }\end{array}$ & & $\checkmark$ & & \\
\hline Pérez-Escamilla et al. $(2015)^{(46)}$ & $\begin{array}{l}\text { 1. Structured blood glucose control programme } \\
\text { 2. Usual care }\end{array}$ & & $\checkmark$ & & \\
\hline Carrasquillo et al. (2017) & $\begin{array}{l}\text { 1. CHW intervention } \\
\text { 2. Enhanced usual care }\end{array}$ & & $\checkmark$ & & \\
\hline Xiang et al. (2017) ${ }^{(18)}$ & $\begin{array}{l}\text { 1. Patient-to-patient education } \\
\text { 2. Control }\end{array}$ & & $\checkmark$ & & \\
\hline Peimani et al. $(2018)^{(57)}$ & $\begin{array}{l}\text { 1. Peer-led diabetes self-management } \\
\text { 2. Education-only control }\end{array}$ & & $\checkmark$ & & \\
\hline Staten et al. $(2004)^{(62)}$ & $\begin{array}{l}\text { 1. Counselling - active control } \\
\text { 2. Counselling and health education } \\
\text { 3. Counselling, health education and PSI }\end{array}$ & & $\checkmark$ & $\checkmark$ & \\
\hline Sreedevi et al. $(2017)^{(53)}$ & $\begin{array}{l}\text { 1. Yoga } \\
\text { 2. Peer Intervention } \\
\text { 3. Control }\end{array}$ & & $\checkmark$ & $\checkmark$ & \\
\hline WC/WHR ( $n$ 18) & & & & & \\
\hline McNabb et al. $(1997)^{(25)}$ & $\begin{array}{l}\text { 1. Experimental group (PSI) } \\
\text { 2. Wait list control }\end{array}$ & $\checkmark$ & & & \\
\hline Heshka et al. (2003) ${ }^{(16)}$ & $\begin{array}{l}\text { 1. Self-help weight loss group } \\
\text { 2. Weight Watchers (PSI) }\end{array}$ & $\checkmark$ & & & \\
\hline Jebb et al. $(2011)^{(31)}$ & $\begin{array}{l}\text { 1. Weight Watchers (PSI) } \\
\text { 2. Standard care }\end{array}$ & $\checkmark$ & & & \\
\hline Katula et al. $(2013)^{(61)}$ & $\begin{array}{l}\text { 1. Diabetes Prevention Program lifestyle weight loss intervention } \\
\text { 2. Enhanced usual care }\end{array}$ & $\checkmark$ & & & \\
\hline Block et al. $(2015)^{(41)}$ & $\begin{array}{l}\text { 1. Alive-PD - a web, Internet, mobile phone intervention } \\
\text { 2. Usual care }\end{array}$ & $\checkmark$ & & & \\
\hline Yang et al. $(2016)^{(33)}$ & $\begin{array}{l}\text { 1. Weight Watchers (PSI) } \\
\text { 2. Nutrition education control }\end{array}$ & $\checkmark$ & & & \\
\hline Debussche et al. (2018) $)^{(24)}$ & $\begin{array}{l}\text { 1. Peer-led self-management education } \\
\text { 2. Usual care }\end{array}$ & $\checkmark$ & & & \\
\hline Verheijden et al. (2004) $)^{(39)}$ & $\begin{array}{l}\text { 1. Web based intervention } \\
\text { 2. Control }\end{array}$ & & $\checkmark$ & & \\
\hline Balcazar et al. $(2010)^{(59)}$ & $\begin{array}{l}\text { 1. CHW education (PSI) } \\
\text { 2. Control }\end{array}$ & & $\checkmark$ & & \\
\hline Parikh et al. (2010) & $\begin{array}{l}\text { 1. Peer-led lifestyle intervention } \\
\text { 2. Delayed intervention control }\end{array}$ & & $\checkmark$ & & \\
\hline
\end{tabular}




\begin{tabular}{|c|c|c|c|c|c|}
\hline \multirow[b]{2}{*}{ Study } & \multirow[b]{2}{*}{ Intervention groups } & \multicolumn{4}{|c|}{ Effect of PSI* } \\
\hline & & $\uparrow$ & $\mathrm{C}$ & I & $\downarrow$ \\
\hline Rosal et al. $(2011)^{(54)}$ & $\begin{array}{l}\text { 1. Tailored diabetes self-management } \\
\text { 2. Usual care }\end{array}$ & & $\checkmark$ & & \\
\hline Islam et al. $(2013)^{(60)}$ & $\begin{array}{l}\text { 1. Project RICE - CHW intervention } \\
\text { 2. Control }\end{array}$ & & $\checkmark$ & & \\
\hline Mash et al. $(2014)^{(23)}$ & $\begin{array}{l}\text { 1. Diabetes education programme } \\
\text { 2. Usual care and education }\end{array}$ & & $\checkmark$ & & \\
\hline Baig et al. $(2015)^{(27)}$ & $\begin{array}{l}\text { 1. Church-based self-management intervention } \\
\text { 2. Usual care and education }\end{array}$ & & $\checkmark$ & & \\
\hline Pérez-Escamilla et al. (2015) ${ }^{(46)}$ & $\begin{array}{l}\text { 1. Structured blood glucose control programme } \\
\text { 2. Usual care }\end{array}$ & & $\checkmark$ & & \\
\hline Staten et al. $(2004)^{(62)}$ & $\begin{array}{l}\text { 1. Counselling - active control } \\
\text { 2. Counselling and health education } \\
\text { 3. Counselling, health education and PSI }\end{array}$ & & $\checkmark$ & $\sqrt{ }$ & \\
\hline Hageman et al. $(2017)^{(36)}$ & $\begin{array}{l}\text { 1. Web intervention } \\
\text { 2. Web intervention + PSI } \\
\text { 3. Web intervention + professional counselling }\end{array}$ & & $\checkmark$ & $\checkmark$ & \\
\hline Sreedevi et al. $(2017)^{(53)}$ & $\begin{array}{l}\text { 1. Yoga } \\
\text { 2. PSI } \\
\text { 3. Control }\end{array}$ & & $\checkmark$ & $\checkmark$ & \\
\hline
\end{tabular}

WC, waist circumference; WHR, waist-to-hip ratio; $\mathrm{CHW}$, community health worker.

*Effect of PSI summarised as: $\uparrow$, PSI more effective than alternative method of support in improving anthropometric outcome; C, no significant difference between PSI and control group in improving anthropometric outcome; I, no significant difference between PSI and intervention group in improving anthropometric outcomes; and $\downarrow$, PSI less effective than other method of support in improving anthropometric outcome.

†Different results for different intervention groups.

‡Different results at different time points.

involved peer supporter as the peer role $(n 9 ; 60 \%)$. It was apparent that a third of studies $(n 2 ; 33 \%)$ that reported no significant difference in PSI for improving overall dietary pattern used a peer case manager as the peer role. This peer role was not used in the five studies that reported that PSI were more effective than alternative methods of support for improving overall dietary pattern. Over one-third of studies that reported that PSI were more effective than alternative methods for improving fat intake ( $n$ 3; 38\%) included health professionals within the PSI delivery, whereas health professionals were not included in the eight studies that reported no significant difference in methods of support for improving fat intake. Half of studies that reported that PSI were more effective than alternative methods of support for improving weight $(n 6 ; 50 \%)$ used a peer supporter as the peer role, whereas only one study reporting no significant difference in methods of support for improving weight $(n 1 ; 10 \%)$ used this peer role. Most studies that reported that PSI were more effective for improving BMI were lay-led ( $n$ 6; $75 \%$ ), whereas almost half of studies that reported no significant difference in methods of support for improving BMI used a CHW ( $n$ 6; 46\%). There were no clear differences in peer roles and types of peer used between studies reporting positive effects of PSI and studies reporting no significant difference between methods of support for improving other dietary or anthropometric outcomes.

Of the forty-eight studies that were peer-led, just over half ( $n$ 33; 69\%) reported that peers were trained to facilitate the intervention. Training programmes ranged in duration from $3 \mathrm{~h}$ to a $36 \mathrm{~h}$ programme delivered over 9 weeks and frequently covered: programme delivery; peer role; group facilitation; behaviour change theory and techniques; adult learning; diet and nutrition principals; disease-specific information; taking health assessments; population-specific information; and supporting skills for supporting individuals such as having empathy and dealing with resistance. In two studies, programme activities were demonstrated via role play and, in a small number of studies ( $n 5 ; 10 \%$ ), individuals undertaking a peer role had the opportunity to practice programme delivery. Few studies $(n 7 ; 15 \%)$ discussed provision of support to individuals undertaking a peer role, but this included: biweekly or monthly meetings with others undertaking a peer role to exchange experiences of delivering a PSI; working alongside an experienced, trained peer; bimonthly supervisory meetings with a health professional; and regular contact with researchers throughout the programme to discuss challenges, answer questions and provide feedback.

Just over one-third of studies ( $n$ 20;37\%) measured fidelity of the PSI delivery. Those that did, measured it through:

1. observation, audio recording or a review of sessions to ensure the structured programme was being followed and performance criteria were being met;

2. supervision of programme delivery by a health professional;

3. notes on PSI sessions kept by individuals undertaking a peer role and contact records with participants; 
Table 4 Characteristics and methodological quality of studies assessing the effect of peer-support interventions (PSI) on dietary behaviour change in adults $(n$ 54)

\begin{tabular}{|c|c|c|c|c|c|c|c|c|c|c|c|c|c|c|}
\hline \multirow[b]{3}{*}{ Study } & \multirow[b]{3}{*}{$\begin{array}{l}\text { Study } \\
\text { location }\end{array}$} & \multicolumn{6}{|c|}{ Participants } & \multirow[b]{3}{*}{$\begin{array}{l}\text { Intervention } \\
\text { groups }\end{array}$} & \multirow{3}{*}{$\begin{array}{c}\text { Duration: } \\
\text { PSI } \\
\text { intensity* } \\
\end{array}$} & \multicolumn{3}{|c|}{ PSI } & \multirow[b]{3}{*}{$\begin{array}{c}\text { No. of } \\
\mathrm{BCT}^{(11)}\end{array}$} & \multirow[b]{3}{*}{ Quality $^{(12)}$} \\
\hline & & \multirow[b]{2}{*}{$\begin{array}{c}\text { No. in } \\
\text { analysis }\end{array}$} & \multirow[b]{2}{*}{$\begin{array}{l}\text { No. in } \\
\text { PSI }\end{array}$} & \multirow[b]{2}{*}{ Gender } & \multicolumn{2}{|c|}{ Age (years) } & \multirow[b]{2}{*}{ Characteristics } & & & \multirow[b]{2}{*}{ Model(s) } & \multirow[b]{2}{*}{ Peer role } & \multirow[b]{2}{*}{ Peer } & & \\
\hline & & & & & Mean & SD & & & & & & & & \\
\hline $\begin{array}{l}\text { Perri et al. } \\
\quad(1987)^{(14)}\end{array}$ & USA & 85 & 32 & $M+F$ & Range: & $21-60$ & Overweight & $\begin{array}{l}\text { 1. Behaviour therapy + PSI } \\
\text { 2. Behaviour therapy + therapist } \\
\text { 3. Behaviour therapy }\end{array}$ & $\begin{array}{l}50 \text { weeks: } \\
\text { M }\end{array}$ & Group & PS & $\begin{array}{l}\text { Health professional } \\
\quad+\text { other participants }\end{array}$ & 3 & Low \\
\hline $\begin{array}{l}\text { Djuric et al. } \\
(2002)^{(15)}\end{array}$ & USA & 39 & 8 & $\mathrm{~F}$ & Range: & $36-70$ & $\begin{array}{l}\text { Mostly White } \\
\text { Obese women with stage } \\
\text { I or II breast cancer }\end{array}$ & $\begin{array}{l}\text { 1. Control } \\
\text { 2. Weight Watchers } \\
\text { 3. Individualised counselling } \\
\text { 4. Individualised counselling \& } \\
\text { Weiaht Watchers }\end{array}$ & 12 months: & Group & PS & Lay individual & - & Moderate \\
\hline $\begin{array}{l}\text { Heshka et al. } \\
(2003)^{(16)}\end{array}$ & USA & 307 & 148 & $M+F$ & $\begin{array}{l}1.44 \\
2.45\end{array}$ & $\begin{array}{l}10.0 \\
10.0\end{array}$ & Overweight/obese & $\begin{array}{l}\text { 1. Self-help weight loss group } \\
\text { 2. Weight Watchers }\end{array}$ & 2 years: & Group & PS & Lay individual & - & High \\
\hline $\begin{array}{l}\text { Pinto et al. } \\
\quad(2013)^{(17}\end{array}$ & USA & 141 & 49 & $M+F$ & 49.7 & 9.2 & $\begin{array}{l}\text { Mostly non-White } \\
\text { Overweight/obese }\end{array}$ & $\begin{array}{l}\text { 1. Professionally led behavioural } \\
\text { weight loss } \\
\text { 2. Weight Watchers } \\
\text { 3. Professionally delivered } \\
\text { behavioural weight loss \& } \\
\text { Weight Watchers }\end{array}$ & $\begin{array}{l}48 \text { weeks: } \\
\text { M }\end{array}$ & Group & PS & Lay individual & - & Low \\
\hline $\begin{array}{l}\text { Xiang et al. } \\
(2017)^{(18)}\end{array}$ & China & 51 & 29 & $M+F$ & $\begin{array}{l}\text { 1. } 53 \cdot 0 \\
\text { 2. } 55 \cdot 4\end{array}$ & $\begin{array}{l}7.3 \\
9.7\end{array}$ & $\begin{array}{l}\text { T2DM with poorly controlled } \\
\text { blood glucose }\end{array}$ & $\begin{array}{l}\text { 1. Patient-to-patient education } \\
\text { 2. Control }\end{array}$ & $\underset{M}{6 \text { months: }}$ & Group & PS & Lay individual & - & High \\
\hline $\begin{array}{l}\text { Cade et al al. } \\
\quad(2009)^{(19)}\end{array}$ & UK & 239 & 112 & $M+F$ & $\begin{array}{l}\text { 1. } 65.4 \\
\text { 2. } 66.2\end{array}$ & $\begin{array}{l}11.6 \\
11.5\end{array}$ & $\begin{array}{l}\text { Mostly white European } \\
\text { T2DM }\end{array}$ & $\begin{array}{l}\text { 1. Diabetes-specific expert } \\
\text { patient programme } \\
\text { 2. Individual dietetic counselling }\end{array}$ & $\begin{array}{l}7 \text { weeks: } \\
M\end{array}$ & Group & PE & Lay individual & - & Low \\
\hline $\begin{array}{l}\text { Lorig et al. } \\
\quad(2009)^{(20)}\end{array}$ & USA & 294 & 161 & $M+F$ & $66 \cdot 7$ & & $\begin{array}{l}\text { Mostly white, non-Hispanic } \\
\text { T2DM }\end{array}$ & $\begin{array}{l}\text { 1. Peer-led diabetes self- } \\
\text { management programme } \\
\text { 2. Usual care }\end{array}$ & $\begin{array}{c}6 \text { weeks: } \\
M\end{array}$ & Group & PE & Lay individual & 5 & Moderate \\
\hline $\begin{array}{l}\text { Parikh et al. } \\
(2010)^{(2 i)}\end{array}$ & USA & 72 & 35 & $M+F$ & 48 & 16.5 & $\begin{array}{l}\text { Mostly Hispanic } \\
\text { Overweight with pre-diabetes }\end{array}$ & $\begin{array}{l}\text { 1. Peer-led lifestyle intervention } \\
\text { 2. Delayed intervention control }\end{array}$ & $\begin{array}{l}12 \text { months: } \\
\mathrm{M}\end{array}$ & Group & PE & Lay individual & - & Moderate \\
\hline $\begin{array}{l}\text { Smith et al. } \\
(2011)^{(22)}\end{array}$ & $\begin{array}{l}\text { Republic of } \\
\text { Ireland }\end{array}$ & 337 & 166 & $M+F$ & $\begin{array}{l}\text { 1. } 66 \cdot 1 \\
\text { 2. } 63.2\end{array}$ & $\begin{array}{l}11.11 \\
11.04\end{array}$ & T2DM & $\begin{array}{l}\text { 1. PSI } \\
\text { 2. Control }\end{array}$ & $\begin{array}{l}2 \text { years: } \\
M\end{array}$ & Group & PE & Lay individual & - & High \\
\hline $\begin{array}{l}\text { Mash et al. } \\
\quad(2014)^{(23)}\end{array}$ & South Africa & 1570 & 710 & $M+F$ & $\begin{array}{l}\text { 1. } 55.8 \\
\text { 2. } 56.4\end{array}$ & $\begin{array}{l}11.5 \\
11.6\end{array}$ & T2DM & $\begin{array}{l}\text { 1. Diabetes education } \\
\text { programme }\end{array}$ & $\begin{array}{l}4 \text { months: } \\
M\end{array}$ & Group & PE & Lay individual & - & Moderate \\
\hline $\begin{array}{l}\text { Debussche } \\
\text { et al. } \\
(2018)^{(24)}\end{array}$ & Africa & 140 & 70 & $M+F$ & $52 \cdot 5$ & 9.8 & T2DM & $\begin{array}{l}\text { 1. Peer-led self-management } \\
\text { education } \\
\text { 2. Usual care }\end{array}$ & $\begin{array}{l}12 \text { months: } \\
\text { M }\end{array}$ & Group & PE & Lay individual & - & Moderate \\
\hline $\begin{array}{l}\text { McNabb et al. } \\
\quad(1997)^{(25)}\end{array}$ & USA & 33 & 15 & $\mathrm{~F}$ & $\begin{array}{l}\text { 1. } 56 \cdot 5 \\
\text { 2. } 56 \cdot 6\end{array}$ & $\begin{array}{l}14.5 \\
13 \cdot 0\end{array}$ & $\begin{array}{l}\text { African-American } \\
\text { Obese women at urban } \\
\text { churches }\end{array}$ & $\begin{array}{l}\text { 1. Experimental group } \\
\text { 2. Wait list control }\end{array}$ & $\begin{array}{l}14 \text { weeks: } \\
\text { M }\end{array}$ & Group & PC & Lay individual & 3 & Low \\
\hline $\begin{array}{l}\text { West et al } \\
(2011)^{(26)}\end{array}$ & USA & 228 & 116 & $M+F$ & $71 \cdot 2$ & $6 \cdot 6$ & $\begin{array}{l}\text { Obese older adults from } \\
\text { senior centres } \\
\text { White }\end{array}$ & $\begin{array}{l}\text { 1. Diabetes prevention } \\
\text { programme } \\
\text { 2. Cognitive training control }\end{array}$ & $\begin{array}{l}12 \text { weeks: } \\
\mathrm{M}\end{array}$ & Group & PC & Lay individual & 7 & High \\
\hline $\begin{array}{l}\text { Baig et al } \\
\quad(2015)^{(27)}\end{array}$ & USA & 84 & 43 & $M+F$ & 53.7 & 11.6 & $\begin{array}{l}\text { Diabetic } \\
\text { Latino }\end{array}$ & $\begin{array}{l}\text { 1. Church-based self- } \\
\text { management intervention } \\
\text { 2. Usual care and education }\end{array}$ & $\begin{array}{c}8 \text { weeks: } \\
\text { M }\end{array}$ & Group & PC & Lay individual & 4 & Moderate \\
\hline $\begin{array}{l}\text { Garrett et al. } \\
\quad(2005)^{(28)}\end{array}$ & USA & 462 & 195 & $M+F$ & Not spe & ecified & $\begin{array}{l}\text { Mostly Caucasian Diabetes } \\
\text { management programme } \\
\text { member }\end{array}$ & $\begin{array}{l}\text { 1. Small group activity } \\
\text { 2. Control }\end{array}$ & $\begin{array}{l}\text { Not stated: } \\
\text { S }\end{array}$ & Group & PE, PS & Lay individual & - & Low \\
\hline $\begin{array}{l}\text { Ackermann } \\
\text { et al. } \\
(2015)^{(29)}\end{array}$ & USA & 509 & 257 & $M+F$ & 51.0 & $12 \cdot 1$ & $\begin{array}{l}\text { African-American/non- } \\
\text { Hispanic White } \\
\text { At risk of T2DM }\end{array}$ & $\begin{array}{l}\text { 1. YMCA adaptation of Diabetes } \\
\text { Prevention Program } \\
\text { 2. Usual care }\end{array}$ & $\begin{array}{l}12 \text { months: } \\
\mathrm{M}\end{array}$ & Group & PE, PS & Lay individual & - & Moderate \\
\hline $\begin{array}{l}\text { Mackinnon } \\
\text { et al. } \\
(2010)^{(30)}\end{array}$ & USA & 424 & 161 & $M+F$ & $40 \cdot 7$ & & $\begin{array}{l}\text { Mostly White, non-Hispanic } \\
\text { Firefighters }\end{array}$ & $\begin{array}{l}\text { 1. Team-based, peer-led } \\
\text { scripted health promotion } \\
\text { 2. One-on-one motivational } \\
\text { interviewing health coaching } \\
\text { 3. Control }\end{array}$ & $\begin{array}{c}2 \text { years: } \\
\mathrm{M}\end{array}$ & Group & PE, PF & Lay individual & 4 & Low \\
\hline $\begin{array}{l}\text { Jebb et al. } \\
\quad(2011)^{(31)}\end{array}$ & $\begin{array}{l}\text { Australia, } \\
\text { Germany, } \\
\text { UK }\end{array}$ & 444 & 230 & $M+F$ & $\begin{array}{l}\text { 1. } 46 \cdot 5 \\
\text { 2. } 48 \cdot 2\end{array}$ & $\begin{array}{l}13.5 \\
12 \cdot 2\end{array}$ & Overweight/obese & $\begin{array}{l}\text { 1. Weight } \\
\text { Watchers } \\
\text { (PSI) } \\
\text { 2. Standard care }\end{array}$ & $\begin{array}{l}12 \text { months: } \\
\mathrm{M}\end{array}$ & Group & PC, PS & Lay individual & - & Moderate \\
\hline
\end{tabular}




\begin{tabular}{|c|c|c|c|c|c|c|c|c|c|c|c|c|c|c|}
\hline \multirow[b]{3}{*}{ Study } & \multirow{3}{*}{$\begin{array}{l}\text { Study } \\
\text { location }\end{array}$} & \multicolumn{6}{|c|}{ Participants } & \multirow[b]{3}{*}{$\begin{array}{l}\text { Intervention } \\
\text { groups }\end{array}$} & \multirow{3}{*}{$\begin{array}{l}\text { Duration: } \\
\text { PSI } \\
\text { intensity* }\end{array}$} & \multicolumn{3}{|c|}{ PSI } & \multirow[b]{3}{*}{$\begin{array}{l}\text { No. of } \\
\mathrm{BCT}^{(11)}\end{array}$} & \multirow[b]{3}{*}{ Quality $y^{12}$} \\
\hline & & \multirow[b]{2}{*}{$\begin{array}{l}\text { No. in } \\
\text { analysis }\end{array}$} & \multirow{2}{*}{$\begin{array}{c}\text { No. in } \\
\text { PSI }\end{array}$} & \multirow[b]{2}{*}{ Gender } & \multicolumn{2}{|c|}{ Age (years) } & \multirow[b]{2}{*}{ Characteristics } & & & \multirow[b]{2}{*}{ Model(s) } & \multirow[b]{2}{*}{ Peer role } & \multirow[b]{2}{*}{ Peer } & & \\
\hline & & & & & Mean & sD & & & & & & & & \\
\hline $\begin{array}{l}\text { Van der Wulp } \\
\text { et al. } \\
(2012)^{(51)}\end{array}$ & Netherlands & 119 & 59 & $M+F$ & \multicolumn{2}{|c|}{ Median: 61} & $\begin{array}{l}\text { Mostly Dutch } \\
\text { T2DM }\end{array}$ & $\begin{array}{l}\text { 1. Peer-led self-management } \\
\text { 2. Control }\end{array}$ & $\begin{array}{l}\text { Not stated: } \\
\text { M }\end{array}$ & $\begin{array}{l}\text { Dyadic } \\
\text { (face-to- } \\
\text { face + telephone) }\end{array}$ & PC & Lay individual & 5 & High \\
\hline $\begin{array}{l}\text { Safford et al. } \\
\quad(2015)^{(52)}\end{array}$ & USA & 360 & 168 & $M+F$ & $60 \cdot 2$ & $12 \cdot 1$ & $\begin{array}{l}\text { Diabetic } \\
\text { African-American }\end{array}$ & $\begin{array}{l}\text { 1. PSI and brief education } \\
\text { 2. Brief education }\end{array}$ & $\begin{array}{l}10 \text { months: } \\
\mathrm{M}\end{array}$ & $\begin{array}{l}\text { Dyadic } \\
\text { (face-to- } \\
\text { face + telephone) }\end{array}$ & PS & Lay individual & - & Moderate \\
\hline $\begin{array}{l}\text { Sreedevi et al. } \\
\qquad(2017)^{(53)}\end{array}$ & India & 99 & 32 & $\mathrm{~F}$ & $\begin{array}{l}\text { 1. } 51.97 \\
\text { 2. } 51.92 \\
\text { 3. } 51.92\end{array}$ & $\begin{array}{l}7.40 \\
8.32 \\
6.57\end{array}$ & Women with T2DM & $\begin{array}{l}\text { 1. Yoga-based intervention } \\
\text { 2. PSI } \\
\text { 3. Control }\end{array}$ & 3 months: & $\begin{array}{l}\text { Dyadic } \\
\text { (face-to- } \\
\text { face + telephone) }\end{array}$ & PC, PS & Lay individual & - & High \\
\hline $\begin{array}{l}\text { Rosal et al. } \\
\qquad(2011)^{(54)}\end{array}$ & USA & 252 & 124 & $M+F$ & \multicolumn{2}{|c|}{$\begin{array}{l}63 \% \text { in age } \\
\text { category } \\
45-64\end{array}$} & $\begin{array}{l}\text { T2DM } \\
\text { Latino }\end{array}$ & $\begin{array}{l}\text { 1. Tailored diabetes self- } \\
\text { management } \\
\text { 2. Usual care }\end{array}$ & $\begin{array}{l}12 \text { months: } \\
\mathrm{M}\end{array}$ & $\begin{array}{l}\text { Hybrid } \\
\text { (group + dyadic face-to- } \\
\text { face) }\end{array}$ & PC & $\begin{array}{l}\text { Lay individuals or nutritionist/ } \\
\text { health educator and lay } \\
\text { individual }\end{array}$ & 8 & High \\
\hline $\begin{array}{l}\text { Campbell et al. } \\
(1999)^{(55)}\end{array}$ & USA & 2519 & 1198 & $M+F$ & \multicolumn{2}{|c|}{$\begin{array}{l}43-64 \\
53 \cdot 8\end{array}$} & $\begin{array}{l}\text { Mostly African-American } \\
\text { Church members }\end{array}$ & $\begin{array}{l}\text { 1. Five--a-day intervention } \\
\text { 2. Control }\end{array}$ & $\begin{array}{l}20 \text { months: } \\
\text { O }\end{array}$ & $\begin{array}{l}\text { Hybrid } \\
\text { (group + dyadic face-to- } \\
\text { face) }\end{array}$ & PE, PS & Lay individual & - & Moderate \\
\hline $\begin{array}{l}\text { Stoddard et al. } \\
\quad(2004)^{(56)}\end{array}$ & USA & 1105 & 600 & $\mathrm{~F}$ & \multicolumn{2}{|l|}{58} & $\begin{array}{l}\text { Mostly White, non-Hispanic } \\
\text { Uninsured and underinsured } \\
\text { women }\end{array}$ & $\begin{array}{l}\text { 1. PSI } \\
\text { 2. Control }\end{array}$ & $\begin{array}{l}12 \text { months: } \\
\mathrm{M}\end{array}$ & $\begin{array}{l}\text { Hybrid } \\
\text { (group + dyadic face-to- } \\
\text { face) }\end{array}$ & $P C, P E, P F$ & Other participants & 2 & Low \\
\hline $\begin{array}{l}\text { Peimani et al. } \\
(2018)^{(57)}\end{array}$ & Iran & 200 & 100 & $M+F$ & $\begin{array}{l}\text { 1. } 59 \cdot 0 \\
\text { 2. } 58.8\end{array}$ & $\begin{array}{l}11 \cdot 3 \\
11.7\end{array}$ & T2DM & $\begin{array}{l}\text { 1. Peer-led diabetes self- } \\
\text { management support } \\
\text { 2. Education-only control }\end{array}$ & 6 months: & $\begin{array}{l}\text { Hybrid } \\
\text { (group +telephone) }\end{array}$ & PS & Lay individual & - & Moderate \\
\hline $\begin{array}{l}\text { Resnicow et al. } \\
(2004)^{(58)}\end{array}$ & USA & 854 & Not stated & $M+F$ & \multicolumn{2}{|l|}{50.6} & $\begin{array}{l}\text { African-American } \\
\text { Church member }\end{array}$ & $\begin{array}{l}\text { 1. Body and Soul intervention } \\
\text { 2. Control }\end{array}$ & $\begin{array}{l}6 \text { months: } \\
0\end{array}$ & $\begin{array}{l}\text { Hybrid } \\
\text { (group + telephone) }\end{array}$ & PC, PE & Lay individual & 1 & Low \\
\hline $\begin{array}{l}\text { Balcazar et al. } \\
(2010)^{(59)}\end{array}$ & USA & 284 & 158 & $M+F$ & \multicolumn{2}{|l|}{54} & Hispanic & $\begin{array}{l}\text { 1. CHW education } \\
\text { 2. Control }\end{array}$ & 4 months: & $\begin{array}{l}\text { Hybrid } \\
\text { (group+telephone) }\end{array}$ & $\mathrm{PE}$ & $\mathrm{CHW}$ & - & Low \\
\hline $\begin{array}{l}\text { Islam et al. } \\
\quad(2013)^{(60)}\end{array}$ & USA & 35 & 21 & $M++F$ & 59.7 & 8.1 & $\begin{array}{l}\text { Korean } \\
\text { At risk of T2DM, aged 18-75 } \\
\text { years }\end{array}$ & $\begin{array}{l}\text { 1. Project RICE - CHW } \\
\text { intervention } \\
\text { 2. Control }\end{array}$ & 6 months: & $\begin{array}{l}\text { Hybrid } \\
\text { (group + telephone) }\end{array}$ & $\mathrm{PC}, \mathrm{PE}$ & $\mathrm{CHW}$ & - & Low \\
\hline $\begin{array}{l}\text { Katula et al. } \\
\quad(2013)^{(61)}\end{array}$ & USA & 261 & 127 & $M+F$ & 57.9 & 9.5 & $\begin{array}{l}\text { Overweight/obese at risk of } \\
\text { T2DM } \\
\text { White }\end{array}$ & $\begin{array}{l}\text { 1. Diabetes Prevention Program } \\
\text { lifestyle weight loss } \\
\text { intervention } \\
\text { 2. Enhanced usual care }\end{array}$ & 2 years: & $\begin{array}{l}\text { Hybrid } \\
\text { (group, dyadic face-to- } \\
\text { face + telephone) }\end{array}$ & PE & $\mathrm{CHW}+$ dietitian & - & Low \\
\hline $\begin{array}{l}\text { Staten et al. } \\
\quad(2004)^{(62)}\end{array}$ & USA & 217 & 67 & $\mathrm{~F}$ & $57 \cdot 2$ & 4.8 & $\begin{array}{l}\text { Hispanic } \\
\text { Underinsured women }\end{array}$ & $\begin{array}{l}\text { 2. Enhanced usual care } \\
\text { 1. Counselling - active control } \\
\text { 2. Counselling and health } \\
\text { education } \\
\text { 3. Counselling, health education } \\
\text { and PSI }\end{array}$ & $\begin{array}{l}12 \text { months: } \\
\mathrm{M}\end{array}$ & $\begin{array}{l}\text { Hybrid } \\
\text { (group, dyadic face-to- } \\
\text { face + telephone) }\end{array}$ & $P C, P E, P F$ & $\mathrm{CHW}$ + nurse & - & Low \\
\hline $\begin{array}{l}\text { Keyserling } \\
\text { et al. } \\
(2008)^{(63)}\end{array}$ & USA & 212 & 106 & $\mathrm{~F}$ & $\begin{array}{l}\text { 1. } 54 \\
\text { 2. } 52\end{array}$ & $\begin{array}{l}0.66 \\
0.64\end{array}$ & $\begin{array}{l}\text { White or African-American } \\
\text { Women in a cancer } \\
\text { detection/CVD prevention } \\
\text { programme }\end{array}$ & $\begin{array}{l}\text { 1. PSI } \\
\text { 2. Control }\end{array}$ & $\begin{array}{l}12 \text { months: } \\
\mathrm{M}\end{array}$ & $\begin{array}{l}\text { Hybrid } \\
\text { (group, dyadic face-to- } \\
\text { face + telephone) }\end{array}$ & $\mathrm{PC}, \mathrm{PF}$ & $\mathrm{CHW}$ + health educator & 9 & Moderate \\
\hline $\begin{array}{l}\text { Spencer et al. } \\
\quad(2011)^{(64)}\end{array}$ & USA & 164 & 72 & $M+F$ & $\begin{array}{l}\text { 1. } 50 \\
\text { 2. } 55\end{array}$ & & $\begin{array}{l}\text { T2DM } \\
\text { African-American and Latino }\end{array}$ & $\begin{array}{l}\text { 1. Diabetes self-management } \\
\text { 2. Delayed control }\end{array}$ & $\underset{M}{6 \text { months: }}$ & $\begin{array}{l}\text { Hybrid } \\
\text { (group, dyadic face-to- } \\
\text { face + telephone) }\end{array}$ & $\mathrm{PC}, \mathrm{PCM}$ & $\mathrm{CHW}$ & - & Moderate \\
\hline $\begin{array}{l}\text { Carrasquillo } \\
\text { et al. } \\
(2017)^{(65)}\end{array}$ & USA & 215 & 111 & $M+F$ & $\begin{array}{l}\text { 1. } 55 \cdot 3 \\
\text { 2. } 55 \cdot 2\end{array}$ & $\begin{array}{l}7.1 \\
6.1\end{array}$ & $\begin{array}{l}\text { Patients of hospital } \\
\text { outpatient clinics with high } \\
\text { HbA1c levels } \\
\text { Latino }\end{array}$ & $\begin{array}{l}\text { 1. PSI } \\
\text { 2. Enhanced usual care }\end{array}$ & $\begin{array}{l}12 \text { months: } \\
\mathrm{M}\end{array}$ & $\begin{array}{l}\text { Hybrid } \\
\text { (group, dyadic face-to- } \\
\text { face + telephone) }\end{array}$ & $P E, P C M$ & $\mathrm{CHW}$ & - & Low \\
\hline $\begin{array}{l}\text { Jolly et al }{ }_{(26)} \\
\qquad(2011)^{(66)}\end{array}$ & UK & 243 & 171 & $M+F$ & $\begin{array}{l}\text { 1. } 50.71 \\
\text { 2. } 48.84 \\
\text { 3. } 49.67\end{array}$ & $\begin{array}{l}14.56 \\
14.91 \\
13.83\end{array}$ & $\begin{array}{l}\text { Mostly white British//rish } \\
\text { Overweight/obese }\end{array}$ & $\begin{array}{l}\text { 1. Weight Watchers (PSI) } \\
\text { 2. Slimming World (PSI) } \\
\text { 3. Exercise minimal intervention }\end{array}$ & $\begin{array}{l}12 \text { weeks: } \\
\mathrm{M}\end{array}$ & $\begin{array}{l}\text { Hybrid } \\
\text { (group + optional dyadic } \\
\text { face-to-face/ } \\
\text { telephone) }\end{array}$ & PE, PS & Lay individual & $\begin{array}{l}\text { 1. } 6 \\
\text { 2. } 8\end{array}$ & Moderate \\
\hline $\begin{array}{l}\text { Allicock et al. } \\
\qquad(2012)^{(67)}\end{array}$ & USA & 562 & 273 & $M+F$ & $51 \cdot 6$ & & $\begin{array}{l}\text { Mostly African-American } \\
\text { Church member }\end{array}$ & $\begin{array}{l}\text { 1. Body and Soul group } \\
\text { 2. Control }\end{array}$ & $\begin{array}{l}6 \text { months: } \\
\mathrm{O}\end{array}$ & $\begin{array}{l}\text { Hybrid(group + optional } \\
\text { dyadic face-to-face/ } \\
\text { telephone) }\end{array}$ & PC, PS & Lay individual & 2 & Low \\
\hline
\end{tabular}

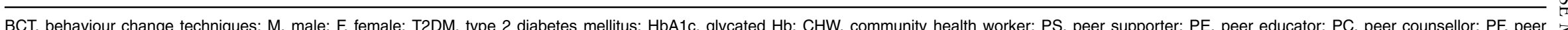

facilitator; PSM, peer case manager.
${ }^{*} \mathrm{PSI}$ intensity summarised as: $\mathrm{M}$, intervention with multiple time points, $\mathrm{S}$, single intervention, $\mathrm{O}$, ongoing intervention. 
4. participant diaries of advice received during the intervention;

5. records of contact between individuals undertaking a peer role and the researchers;

6. focus groups/interviews with individuals undertaking a peer role, participants and the researchers about their experience of the intervention; and

7. a participant questionnaire regarding the delivered intervention.

The theoretical basis of the PSI was stated in just over half of studies ( $n$ 28; 52\%). The most commonly used theories in included studies were Social Cognitive Theory $^{(68)}$ ( $n$ 11; 39\%), the Transtheoretical Model ${ }^{(69)}$ ( $n$ 8; $29 \%)$ and Social Support Theory ${ }^{(70)}(n 7 ; 25 \%)$, but it is not known if different or any theories were used in studies where theory was not reported. A summary of BCT used to encourage dietary behaviour change is shown in Table 4 . The use of BCT was clearly described in twentyfive studies (46\%). It is therefore not known in some studies if techniques were used, or if further techniques to those reported were used. Of the twenty-five studies that clearly described BCT, over one-quarter of these studies ( $n 7 ; 28 \%$ ) reported that the PSI was more effective than alternative interventions and/or a control for improving dietary behaviour change outcomes. These seven studies used a mean of six BCT. The most frequently used BCT were behavioural goal setting (used by $n$ 5; 71\%), prompting self-monitoring (used by $n$ 4; $57 \%$ ) and provision of feedback (used by $n$ 4; $57 \%$ ). The eighteen remaining studies that clearly described BCT reported mixed results ( $n$ 13; 52\%) or no significant difference between the PSI and alternative interventions and/or a control ( $n$ 5; 20\%). The five studies that reported no significant difference used a mean of three BCT, with frequently incorporated techniques being planning for social support/social change ( $n$ 3; $60 \%$ ) and barrier identification/problem solving ( $n$ 3; 60\%). Studies that included behaviour change theory ( $n$ 28; 52\%) reported positive effects of PSI ( $n 7 ; 25 \%)$, no significant difference between methods of support ( $n$ 9; $32 \%$ ) and mixed results ( $n$ 12; $43 \%)$. Similarly, studies that did not discuss inclusion of behaviour change theory ( $n 26 ; 48 \%$ ) also reported positive effects of PSI ( $n 8 ; 31 \%)$, no significant difference between methods of support ( $n 8 ; 31 \%)$ and mixed results ( $n$ 10; 38\%). Considering the main behaviour change theories used in the twenty-eight studies that discussed inclusion of behaviour change theory, namely Social Cognitive Theory ${ }^{(68)}$ ( $n$ 11; 39\%), the Transtheoretical Model $^{(69)}\left(n\right.$ 8; 29\%) and Social Support Theory ${ }^{(70)}$ ( $n$ 7; $25 \%)$, there were no clear differences in PSI effectiveness with inclusion of each of these theories.

Intervention periods ranged from 6 weeks to 30 months. The duration of over half of the fifteen studies reporting a positive effect of PSI was $\geq 1$ year ( $n 9 ; 60 \%$ ), whereas the duration of over half of the seventeen studies that reported no significant difference between PSI and alternative methods of support and/or a control ( $n 11 ; 65 \%$ ) was $<1$ year. There were no obvious differences in study intensity between studies reporting a positive effect of PSI on dietary behaviour change and studies reporting no significant difference or mixed results.

Most studies took place in the USA ( $n$ 41; 76\%). The remaining studies took place in China ( $n 2 ; 4 \%$ ), the UK ( $n 2 ; 4 \%$ ), Republic of Ireland ( $n 1 ; 2 \%)$, the Netherlands ( $n 1 ; 2 \%)$, Canada ( $n 1 ; 2 \%)$, Australia ( $n 1 ; 2 \%)$, India ( $n 1$; $2 \%)$, Iran ( $n 1 ; 2 \%)$, South Africa $(n 1 ; 2 \%)$ and Mali ( $n 1$; $2 \%$ ), while one study ( $2 \%$ ) took place across three locations (Australia, Germany and the UK). Of the forty-one studies that reported participant ethnicity, the ethnicities most commonly included by studies were White Americans ( $n$ 10; 24\%), African-Americans ( $n$ 8; $20 \%$ ) and Hispanics ( $n 5 ; 12 \%$ ). Studies including a mostly Hispanic or White American population mostly reported mixed results. Of studies including a mostly African-American population, studies reported different findings on the effectiveness of PSI for improving dietary behaviour: four studies (50\%) reported that the PSI was more effective than alternative methods of support, two reported mixed results (25\%) and two reported no significant difference between methods of support (25\%). A range of population groups were used across studies. The main population groups included individuals with type 2 diabetes mellitus ( $n$ 20; 37\%) and overweight/obese individuals ( $n$ 14; $26 \%$ ). Considering the two main population groups included in the study, studies that included overweight/obese individuals ( $n$ 14; $26 \%$ ) all reported positive ( $n 5 ; 36 \%$ ) or mixed effects of PSI ( $n$ ); $64 \%$ ) on dietary behaviour change outcomes. Studies that included individuals with type 2 diabetes mellitus ( $n$ 20; $37 \%$ ) mainly reported no significant difference ( $n 11 ; 55 \%)$ or mixed results ( $n 6 ; 30 \%$ ). Considering studies measuring the effect of PSI on fat intake, studies that reported no significant difference ( $n$ 8; 44\%) largely included individuals with type 2 diabetes mellitus ( $n 5 ; 63 \%$ ), whereas in studies that reported positive results ( $n 8 ; 44 \%$ ), population groups varied. Studies that reported PSI to be more effective for improving weight ( $n 12 ; 44 \%$ ) or that reported mixed results ( $n$ 5; 19\%) mostly included overweight/ obese individuals ( $n 12 ; 71 \%$ ), whereas half of studies that reported no significant difference between methods of support for improving weight ( $n$ 10; 37\%) included a sample of individuals with type 2 diabetes mellitus ( $n$ 5; $50 \%$ ). Studies reporting that PSI were more effective than a control for improving waist and hip measurements ( $n$ 7; $39 \%$ ) largely included overweight/obese individuals ( $n$ 5; $71 \%)$, whereas studies that reported no significant difference ( $n$ 11; 61\%) included various populations. There were no clear differences in populations included between studies reporting positive effects of PSI and studies reporting no significant difference between methods of support for improving other dietary or anthropometric outcomes. 


\section{Risk of bias}

As shown in Table 4, over one-third of included studies were classified as low ( $n$ 19; 35\%) or moderate ( $n$ 21; $39 \%$ methodological quality and approximately onequarter of studies were classified as high methodological quality ( $n$ 14; 26\%). Half of included studies ( $n 27 ; 50 \%$ ), described the method of randomisation and used an appropriate method (e.g. computer-generated randomisation sequence). Double blinding was not reported by any studies as it is generally not feasible to blind participants in the delivery of PSI; however, participants were blinded to randomisation in one web-based study and participants were blinded to the study hypothesis in another. In a small number of studies outcome assessment was blinded ( $n 9 ; 17 \%$ ) or the research team was blinded to randomisation $(n 6 ; 11 \%)$. Less than half of included studies ( $n$ 22; 41\%) recorded the number of participants that withdrew from the study and the reasons for withdrawal. Studies that were classified as low methodological quality ( $n$ 19; 35\%) did not describe the method of randomisation, use double blinding, or record the number of participants who withdrew from the study and the reasons for withdrawal.

More studies classified as high methodological quality reported positive or mixed effects of PSI on dietary behaviour change outcomes ( $n 8 ; 57 \%$ ) than reporting no significant difference between methods of support $(n 6$; $43 \%$ ). This is consistent with overall results from all studies on the effect of PSI on dietary behaviour change.

\section{Discussion}

The present review aimed to assess the effectiveness of PSI for encouraging dietary behaviour change in adults and to consider intervention characteristics that might be linked with effectiveness. Findings were inconsistent and therefore do not provide clear evidence supporting the effect of PSI on dietary behaviour, while there is also insufficient evidence to make firm conclusions on what characteristics of PSI are linked with effectiveness.

\section{Effect of peer-supported interventions on dietary behaviour change}

Overall, the effect of PSI on dietary behaviour change varied; however, the majority of studies reported that PSI were more effective than alternative interventions and/or a control for improving outcome(s) related to dietary behaviour change or reported mixed results, while $31 \%$ found no significant benefits of PSI. Examination of PSI details highlighted that most studies were group-based or used a combination of models and were lay-led.

Across studies, there was limited detail on intervention development, content and process evaluation, as recommended in the Medical Research Council's framework for development and evaluation of complex interventions to improve health ${ }^{(71)}$. Only one study ${ }^{(24)}$ was reported according to the Template for Intervention Description and Replication (TIDieR) checklist ${ }^{(72)}$, which enables better reporting of intervention details. Several studies did not describe the intensity of the PSI. Just under half of studies that used peers to facilitate the intervention did not mention if training was provided for undertaking this role and limited information was available from studies that reported including training. Few studies discussed provision of support for individuals undertaking a peer role and just over one-third measured the fidelity of the delivered PSI. It is therefore possible that the PSI offered may not have been optimal for encouraging dietary behaviour change. Future interventions assessing the effect of PSI on dietary behaviour change should clearly describe these processes using the available guidance for intervention reporting to enable better detection of factors that may contribute to the effectiveness of PSI to encourage dietary behaviour change.

There was high heterogeneity between studies. Previous reviews considering the effect of PSI on health behaviours have also reported heterogeneity between studies $^{(3,5,73)}$. The mixed evidence found may be due to variation in study characteristics. Sample sizes varied widely. Some studies including small sample sizes may have had insufficient power to detect change in dietary behaviour. Studies also measured different outcomes which are difficult to compare. It may be easier to increase fruit and vegetable intake, for example, than to lose weight or decrease fat intake. Heterogeneity in results, however, was observed within most outcomes.

\section{Effect of peer-supported intervention characteristics on dietary behaviour change}

Study characteristics linked with PSI effectiveness for achieving dietary behaviour change, based on studies assessing anthropometric outcomes, included use of a weight management intervention and targeting an overweight/obese sample. This group may have greater motivation for behaviour change than the general population. Further research is needed to determine if PSI are particularly effective for certain population groups or dietary related outcomes. It is possible that the ethnicity of populations included in studies may impact the effectiveness of PSI. However, as shown in the present review, studies conducted with the same ethnic groups reported different effects of PSI and as small numbers of studies included each ethnic group, further research is needed to investigate this. Studies used different intervention models and types of peer to facilitate the intervention, which may differently affect dietary behaviour. More studies using a group PSI model were effective than studies using other PSI models. Evidence from systematic reviews considering the effect of PSI on health behaviours has found no 
significant effect of PSI models on behaviour change ${ }^{(5)}$ or suggested that dyadic support may be most effective ${ }^{(8)}$. It has been stated that, based on current evidence, no one PSI model is superior to another for achieving change ${ }^{(3)}$ and different models may be suited to different populations and settings ${ }^{(74)}$, or a combination of models may offer optimal support ${ }^{(22)}$. Lay-led support was linked with PSI effectiveness in the present review. Some studies used other participants, who were not trained for delivering a PSI, or included a health professional in the PSI team, which changes the reciprocal PSI relationship ${ }^{(75)}$. Studies assessing effectiveness of PSI on fat intake, however, were linked with effectiveness where health professionals were included in the PSI team. It is possible that professional guidance is helpful for making this type of dietary behaviour change. Use of a peer supporter was also linked with PSI effectiveness in the current review, which is consistent with a previous review ${ }^{(8)}$. Process evaluations of PSI should include determining an optimal peer role and the key characteristics and skills required to undertake it ${ }^{(76)}$. Intervention duration ranged from 6 weeks to 30 months. Over half of studies reporting positive effects of PSI ( $n$ 9; $60 \%$ ) were at least 1 year in duration, which may imply that a longer PSI duration is needed to effectively establish dietary change in adults. Minimal research exists that considers the ideal length of time required for successful dietary interventions.

There were no clear differences in PSI effectiveness with use of different behaviour change theories. BCT used in interventions shown to be effective in the present review included behavioural goal setting, prompting selfmonitoring and provision of feedback. These BCT have been associated with improved dietary behaviour in other systematic reviews ${ }^{(77)}$ and may be useful to incorporate into future PSI to successfully facilitate dietary behaviour change. Studies reporting positive effects of PSI employed more BCT to target dietary behaviour change than studies reporting no effect. Previous research, however, suggests that the number of BCT employed in interventions does not have an effect on behaviour change ${ }^{(78)}$, therefore it may be more important to consider the appropriate $\mathrm{BCT}$ to include rather than the number. Studies included in the present review used different combinations of BCT. The optimal number and type of BCT to use are likely to depend on the type of dietary behaviour change and the population being investigated. Further research is needed to determine optimal combinations of BCT and behaviour change theories for use in PSI to encourage dietary behaviour change in adults. This will be determined by better reporting of intervention development, content and process evaluation and by using feasibility and pilot studies to address issues in study design.

The implementation of well-designed and welldescribed PSI interventions to encourage dietary behaviour change will provide an evidence base for further exploration of study characteristics linked with effectiveness in encouraging dietary behaviour change in adults.

\section{Limitations}

In the interpretation of these findings, it is important to consider the following limitations at the review level. First, considering the search stage, the incorporation of the different search terms associated with PSI yielded a large volume of studies that did not necessarily incorporate a PSI. This, however, helped to ensure that key studies were captured. The search did not include grey literature; therefore some studies may have been missed and the impact of publication bias could not be determined due to the heterogeneity of results. The search also excluded study designs other than randomised controlled trials. Future reviews of PSI could consider inclusion of other study designs to examine the additional information that they may provide. Second, title and abstract screening and data extraction were conducted by one reviewer; however, two reviewers independently screened all potentially relevant full texts and data extraction was checked by a second reviewer. Third, owing to the methodological diversity of included studies in terms of design and outcomes, the data could not be meta-analysed. However, the narrative synthesis undertaken was implemented rigorously to reduce the potential of bias. At the study level, first it should be considered that over one-third of studies were classified as low methodological quality, largely because blinding is generally not feasible in PSI. Second, measurement of dietary behaviour change outcomes may have been subject to bias as self-report methods of dietary assessment were used ${ }^{(79-81)}$ rather than objective markers of actual change such as nutritional biomarkers, and it was not clear in all studies if anthropometric measurements were taken using a standardised approach.

\section{Conclusion}

The majority of studies (69\%) reported that PSI were more effective than alternative methods of support or a control for improving dietary behaviour change related outcome(s) or reported mixed results, and $31 \%$ found no significant benefits of PSI. As evidence was mixed, however, the effect of PSI in encouraging dietary behaviour change in adults is not clear. The present systematic review is the first on the effect of PSI on dietary behaviour in adults and has demonstrated that there is currently insufficient evidence to recommended incorporation of PSI into public health policy and practice for encouraging dietary behaviour change in adults. Further well-designed interventions need to be undertaken to build an evidence base for the use of PSI for achieving dietary behaviour change. The review has highlighted specific details needed in future research to advance this field. Descriptive reporting of intervention 
development and content (including the theoretical basis, BCT used, intervention intensity and peer training and support needs) is needed. Researchers should also report process evaluations of intervention elements that worked or not and ideally examine how behaviour change theory and techniques are linked with effectiveness via mediator and moderator analysis.

\section{Acknowledgements}

Acknowledgements: The research team wishes to acknowledge Professor Mike Clarke for his useful advice regarding the review search strategy. Financial support: This research was supported by PhD studentship funding provided by the Department of Education and Learning and funding from the National Prevention Research Initiative (NPRI; http://www.npri.org.uk) (grant number MR/ J000388/1). NPRI funding partners (in alphabetical order) were: Alzheimer's Research Trust; Alzheimer's Society; Biotechnology and Biological Sciences Research Council; British Heart Foundation; Cancer Research UK; Chief Scientist Office, Scottish Government Health Directorate; Department of Health; Diabetes UK; Economic and Social Research Council; Health and Social Care Research and Development Division of the Public Health Agency (HSC R\&D Division); Medical Research Council; The Stroke Association; Wellcome Trust; Welsh Assembly Government; and World Cancer Research Fund. The funders had no role in the design, analysis or writing of this article. Conflict of interest: None. Authorship: S.E.M. and C.T.M. devised the search strategy. S.E.M. conducted the database searches, study selection, data extraction, data synthesis and drafted the manuscript, and M.M. provided substantial assistance with this process. J.V.W. and M.C.M. designed the review and provided guidance throughout the review process. J.V.W. assisted in study selection and data extraction. All authors critically reviewed drafts of the manuscript. Ethics of human subject participation: Not applicable.

\section{Supplementary material}

To view supplementary material for this article, please visit https://doi.org/10.1017/S1368980018003294

\section{References}

1. World Health Organization \& Food and Agriculture Organization of the United Nations (2003) Diet, nutrition and the prevention of chronic diseases. Report of a Joint WHO/FAO Consultation. WHO Technical Report Series no. 916. http:// apps.who.int/iris/bitstream/10665/42665/1/WHO_TRS_916. pdf?ua=1 (accessed October 2017).

2. Heisler M (2007) Overview of PS models to improve diabetes self-management and clinical outcomes. Diabet Spectr 20, 214-221.
3. Dale JR, Williams SM \& Bowyer V (2012) What is the effect of peer support on diabetes outcomes in adults? A systematic review. Diabet Med 29, 1361-1377.

4. Zhang X, Yang S, Sun K et al. (2016) How to achieve better effect of peer support among adults with type 2 diabetes: a meta-analysis of randomized clinical trials. Patient Educ Couns 99, 186-197.

5. Webel AR, Okonsky J, Trompeta J et al. (2010) A systematic review of the effectiveness of peer-based interventions on health-related behaviors in adults. Am J Public Health 100, 247-253.

6. Best KL, Miller WC, Eng JJ et al. (2016) Systematic review and meta-analysis of peer-led self-management programs for increasing physical activity. Int J Behav Med 23, $527-538$

7. Dennis C-L (2003) PS within a health care context: a concept analysis. Int J Nurs Stud 40, 321-332.

8. Ramchand R, Ahluwalia SC, Xenakis L et al. (2017) A systematic review of peer-supported interventions for health promotion and disease prevention. Prev Med 101, 156-170.

9. Webb TL, Jospeh J, Yardley L et al. (2010) Using the internet to promote health behavior change: a systematic review and meta-analysis of the impact of theoretical basis, use of behavior change techniques, and mode of delivery on efficacy. J Med Internet Res 12, e4.

10. Moher D, Liberati A, Tetzlaff J et al. (2009) Preferred reporting items for systematic reviews and meta-analyses: the PRISMA statement. BMJ 339, b2535.

11. Michie S, Ashford S, Sniehotta FF et al. (2011) A refined taxonomy of behaviour change techniques to help people change their physical activity and healthy eating behaviours: the CALORE taxonomy. Psychol Health 26, 1479-1498.

12. Jadad AR, Moore RA, Carroll D et al. (1996) Assessing the quality of reports of randomized clinical trials: is blinding necessary? Control Clin Trials 17, 1-12.

13. Popay J, Roberts H, Sowden A et al. (2006) Guidance on the Conduct of Narrative Synthesis in Systematic Reviews: A Product of the ESRC Methods Programme, pp. 11-16. Lancaster: University of Lancaster

14. Perri MG, Lauer JB, Yancey DZ et al. (1987) Effects of peer support and therapist contact on long-term weight loss. $J$ Consult Clin Psychol 55, 615-617.

15. Djuric Z, DiLaura NM, Jenkins I et al. (2002) Combining weight-loss counseling with the weight watchers plan for obese breast cancer survivors. Obes Res 10, 657-665.

16. Heshka S, Anderson JW, Atkinson RL et al. (2003) Weight loss with self-help compared with a structured commercial program: a randomized trial. JAMA 289, 1792-1798.

17. Pinto AM, Fava JL, Hoffmann DA et al. (2013) Combining behavioral weight loss treatment and a commercial program: a randomized clinical trial. Obesity (Silver Spring) 21, 673-680.

18. Xiang Y, Luo P, Cai X, et al. (2017) Results of a pilot study of patient-to-patient education strategy on self-management among glycemic uncontrolled patients with diabetes. Patient Prefer Adherence 11, 787-793.

19. Cade JE, Kirk SFL, Nelson P et al. (2009) Can peer educators influence healthy eating in people with diabetes? Results of a randomized controlled trial. Diabet Med 26, 1048-1054.

20. Lorig K, Ritter PL, Villa FJ et al. (2009) Community-based peer-led diabetes self-management: a randomized trial. Diabetes Educ 35, 641-651.

21. Parikh P, Simon EP, Fei K et al. (2010) Results of a pilot diabetes prevention intervention in East Harlem, New York City: Project HEED. Am J Public Health 100, Suppl. 1, S232-S239.

22. Smith SM, Paul G, Kelly A et al. (2011) Peer support for patients with type 2 diabetes: cluster randomised controlled trial. BMJ 342, d715. 
23. Mash RJ, Rhode H, Zwarenstein M et al. (2014). Effectiveness of a group diabetes education programme in underserved communities in South Africa: a pragmatic cluster randomized controlled trial. Diabet Med 31, 987-993.

24. Debussche X, Besançon S, Balcou-Debussche $\mathrm{M}$ et al. (2018) Structured peer-led diabetes self-management and support in a low-income country: the ST2EP randomised controlled trial in Mali. PLoS One 13, e0191262.

25. McNabb W, Quinn M, Kerver J et al. (1997) The PATHWAYS church-based weight loss program for urban AfricanAmerican women at risk for diabetes. Diabetes Care $\mathbf{2 0}$, 1518-1523.

26. West DS, Bursac Z, Cornell CE et al. (2011) Lay health educators translate a weight-loss intervention in senior centers: a randomized controlled trial. Am J Prev Med $\mathbf{4 1}$, 385-391.

27. Baig AA, Benitez A, Locklin CA et al. (2015) Picture Good Health: a church-based self-management intervention among Latino adults with diabetes. J Gen Intern Med 30 , 1481-1490.

28. Garrett N, Hageman CM, Sibley SD et al. (2005) The effectiveness of an interactive small group diabetes intervention in improving knowledge, feeling of control, and behavior. Health Promot Pract 6, 320-328.

29. Ackermann RT, Liss DT, Finch EA et al. (2015) A randomized comparative effectiveness trial for preventing type 2 diabetes. Am J Public Health 105, 2328-2334.

30. MacKinnon DP, Elliot DL, Thoemmes F et al. (2010) Longterm effects of a worksite health promotion program for firefighters. Am J Health Behav 34, 695-706.

31. Jebb SA, Ahern AL, Olson AD et al. (2011) Primary care referral to a commercial provider for weight loss treatment versus standard care: a randomised controlled trial. Lancet 378, 1485-1492.

32. Robinson-Whelen S, Hughes RB, Taylor HB et al. (2006) Improving the health and health behaviors of women aging with physical disabilities: a peer-led health promotion program. Womens Health Issues 16, 334-345.

33. Yang Z, Yu Z, Jiang Y et al (2016) Evaluation of a community-based behavioral weight loss program in Chinese adults: a randomized controlled trial. Obesity (Silver Spring) 24, 1464-1470.

34. Toobert DJ, Strycker LA, Barrera M Jr et al. (2011) Outcomes from a multiple-risk-factor diabetes trial for Latinas: Viva Bien! Ann Behav Med 41, 310-232.

35. Toobert DJ, Glasgow RE, Strycker LA et al. (2007) Long-term effects of the Mediterranean lifestyle program: a randomized clinical trial for postmenopausal women with type 2 diabetes. Int J Behav Nutr Phys Act 4, 1.

36. Hageman PA, Pullen CH, Hertzog M et al. (2017) Webbased interventions alone or supplemented with peer-led support or professional email counseling for weight loss and weight maintenance in women from rural communities: results of a clinical trial. J Obes 2017, 1602627.

37. Winett RA, Anderson ES, Wojcik JR et al. (2007) Guide to health: nutrition and physical activity outcomes of a grouprandomized trial of an Internet-based intervention in churches. Ann Behav Med 33, 245-255.

38. Glasgow RE, Boles SM, McKay G et al. (2003) The D-Net diabetes self-management program: long-term implementation, outcomes, and generalization results. Prev Med 36, 410-419.

39. Verheijden M, Bakx JC, Akkermans R et al. (2004) Web-based targeted nutrition counselling and social support for patients at increased cardiovascular risk in general practice: randomized controlled trial. J Med Internet Res $\mathbf{6}$, e44.

40. Sternfeld B, Block C, Quesenberry CP Jr et al. (2009) Improving diet and physical activity with ALIVE, a worksite randomized trial. Am J Prev Med 36, 475-483.
41. Block G, Azar KM, Romanelli RJ, et al. (2015) Diabetes prevention and weight loss with a fully automated behavioral intervention by email, web, and mobile phone: a randomized controlled trial among persons with prediabetes. J Med Internet Res 17, e240.

42. Jane M, Hagger M, Foster J et al. (2017) Effects of a weight management program delivered by social media on weight and metabolic syndrome risk factors in overweight and obese adults: a randomised controlled trial. PLoS One 12, e0178326.

43. Chang M-W, Brown R \& Nitzke S (2017) A communitybased intervention program's effects on dietary intake behaviors. Obesity (Silver Spring) 25, 2055-2061.

44. Corkery E, Palmer C, Foley ME et al. (1997) Effect of a bicultural community health worker on completion of diabetes education in a Hispanic population. Diabetes Care 20, 254-257.

45. Rothschild SK, Martin MA, Swider SM et al. (2014) Mexican American trial of community health workers: a randomized controlled trial of a community health worker intervention for Mexican Americans with type 2 diabetes mellitus. $\mathrm{Am} \mathrm{J}$ Public Health 104, 1540-1548.

46. Pérez-Escamilla R, Damio G, Chhabra J et al. (2015) Impact of a community health workers-led structured program on blood glucose control among Latinos with type 2 diabetes: the DIALBEST Trial. Diabetes Care 38, 197-205.

47. Prezio EA, Cheng D, Balasubramanian BA et al. (2013) Community Diabetes Education (CoDE) for uninsured Mexican Americans: a randomized controlled trial of a culturally tailored diabetes education and management program led by a community health worker. Diabetes Res Clin Pract 100, 19-28.

48. Lutes LD, Cummings DM, Littlewood K et al. (2017) A community health worker-delivered intervention in African American women with type 2 diabetes: a 12-month randomized trial. Obesity (Silver Spring) 25, 1329-1335.

49. Gary TL, Bone LR, Hill MN et al. (2003) Randomized controlled trial of the effects of nurse case manager and community health worker interventions on risk factors for diabetes-related complications in urban African Americans. Prev Med 37, 23-32.

50. Babamoto KS, Sey KA, Camilleri AJ et al. (2009) Improving diabetes care and health measures among Hispanics using community health workers: results from a randomized controlled trial. Health Educ Behav 36, 113-126.

51. Van der Wulp I, de Leeuw JRJ, Gorter KJ et al. (2012) Effectiveness of peer-led self-management coaching for patients recently diagnosed with type 2 diabetes mellitus in primary care: a randomized controlled trial. Diabet Med 29, e390-e397.

52. Safford MM, Andreae S, Cherrington AL et al. (2015) Peer coaches to improve diabetes outcomes in rural Alabama: a cluster randomized trial. Ann Fam Med 13, Suppl. 1, S18-S26.

53. Sreedevi A, Gopalakrishnan UA, Karimassery Ramaiyer S et al. (2017) A randomized controlled trial of the effect of yoga and peer support on glycaemic outcomes in women with type 2 diabetes mellitus: a feasibility study. $B M C$ Complement Altern Med 17, 100.

54. Rosal MC, Ockene IS, Restrepo A et al. (2011) Randomized trial of a literacy-sensitive, culturally tailored diabetes selfmanagement intervention for low-income Latinos: Latinos en control. Diabetes Care 34, 838-844.

55. Campbell MK, Demark-Wahnefried W, Symons $\mathrm{M}$ et al. (1999) Fruit and vegetable consumption and prevention of cancer: the Black Churches United for Better Health project. Am J Public Health 89, 1390-1396.

56. Stoddard AM, Palombo R, Troped PJ et al. (2004) Cardiovascular disease risk reduction: the Massachusetts WISEWOMAN project. $J$ Womens Health 13, 539-546. 
57. Peimani M, Monjazebi F, Ghodssi-Ghassemabadi R et al. (2018). A peer support intervention in improving glycemic control in patients with type 2 diabetes. Patient Educ Couns 101, 460-466.

58. Resnicow K, Campbell MK, Carr C et al. (2004) Body and Soul. A dietary intervention conducted through AfricanAmerican churches. Am J Prev Med 27, 97-105.

59. Balcazar HG, de Heer H, Rosenthal L et al. (2010) A promotores de salud intervention to reduce cardiovascular disease risk in a high-risk Hispanic border population, 2005-2008. Prev Chronic Dis 7, A28.

60. Islam NS, Zanowiak JM, Wyatt LC et al. (2013) A randomized-controlled, pilot intervention on diabetes prevention and healthy lifestyles in the New York City Korean community. J Community Health 38, 1030-1041.

61. Katula JA, Vitolins MZ, Morgan TM et al. (2013) The Healthy Living Partnerships to Prevent Diabetes Study: 2-year outcomes of a randomized controlled trial. Am J Prev Med 44, 4 Suppl. 4, S324-S332.

62. Staten LK, Gregory-Mercado KY, Ranger-Moore J et al. (2004) Provider counseling, health education, and community health workers: the Arizona WISEWOMAN project. $J$ Womens Health 13, 547-556.

63. Keyserling TC, Samuel Hodge CD, Jilcott SB et al. (2008) Randomized trial of a clinic-based, community-supported, lifestyle intervention to improve physical activity and diet: the North Carolina enhanced WISEWOMAN project. Prev Med 46, 499-510.

64. Spencer MS, Rosland A-M, Kieffer EC et al. (2011) Effectiveness of a community health worker intervention among African American and Latino adults with type 2 diabetes: a randomized controlled trial. Am J Public Health 101, 2253-2260.

65. Carrasquillo O, Lebron C, Alonzo Y et al. (2017) Effect of a community health worker intervention among Latinos with poorly controlled type 2 diabetes: the Miami healthy heart initiative randomized clinical trial. JAMA Intern Med 177, 948-954.

66. Jolly K, Lewis A, Beach J et al. (2011) Comparison of range of commercial or primary care led weight reduction programmes with minimal intervention control for weight loss in obesity: Lighten Up randomised controlled trial. BMJ $\mathbf{3 4 3}$, d6500.

67. Allicock M, Campbell MK, Valle CG et al. (2012) Evaluating the dissemination of Body \& Soul, an evidence-based fruit and vegetable intake intervention: challenges for dissemination and implementation research. J Nutr Educ Behav 44, 530-538.

68. Bandura A (1998) Health promotion from the perspective of social cognitive theory. Psychol Health 13, 623-649.

69. Prochaska JO, DiClemente CC \& Norcross JC (1992) In search of how people change: applications to addictive behaviors. Am Psychol 47, 1102-1114.

70. Cohen S, Gottlieb B \& Underwood L (2000) Social relationships and health. In Social Support Measurement and Intervention, pp. 3-28 [S Cohen, L Underwood and B Gottlieb, editors]. New York: Oxford University Press.

71. Campbell NC, Murray E, Darbyshire J et al. (2007) Designing and evaluating complex interventions to improve health care. BMJ 334, 455-459.

72. Hoffman TC, Glasziou PP, Boutron I et al. (2014) Better reporting of interventions: template for intervention description and replication (TIDieR) checklist and guide. BMJ 348, g1687.

73. Foster G, Taylor SJ, Eldridge SE et al. (2007) Selfmanagement education programmes by lay leaders for people with chronic conditions. Cochrane Database Syst Rev issue 4, CD005108.

74. Fisher EB, Earp JA, Maman S et al. (2010) Cross-cultural and international adaptation of peer support for diabetes management. Fam Pract 27, i6-i16.

75. MacPherson SL, Joseph D \& Sullivan E (2004) The benefits of peer support with diabetes. Nurs Forum 39, 4-12.

76. Pérez-Escamilla R, Hromi-Fiedler A, Vega-López S et al. (2008) Impact of peer nutrition education on dietary behaviors and health outcomes among Latinos: a systematic literature review. J Nutr Educ Behav 40, 208-225.

77. Greaves CJ, Sheppard KE, Abraham C et al. (2011) Systematic review of reviews of intervention components associated with increased effectiveness in dietary and physical activity interventions. BMC Public Health 11, 119.

78. Michie S, Abraham C, Whittington C et al. (2009) Effective techniques in healthy eating and physical activity interventions: a meta-regression. Health Psychol 28, 690-701.

79. Cade J, Thompson R, Burley V et al. (2001) Development, validation and utilisation of food-frequency questionnaires a review. Public Health Nutr 5, 567-587.

80. Tamaro S, Hudson TS, Forman MR et al. (2006) Dietary fiber intake: assessing the degree of agreement between food frequency questionnaires and 4-d food records. J Am Coll Nutr 25, 370-381.

81. Ambrosini GL, de Klerk NH, O'Sullivan TA et al. (2009) The reliability of a food frequency questionnaire for use among adolescents. Eur J Clin Nutr 63, 1251-1259. 